\title{
Streamlined and Accurate Gesture Recognition with Penny Pincher
}

\author{
Eugene M. Taranta II ${ }^{\mathrm{a}, *}$, Andrés V. González ${ }^{\mathrm{a}}$, Joseph J. LaViola Jr. ${ }^{\mathrm{a}}$ \\ ${ }^{a}$ Department of EECS, University of Central Florida, 4000 Central Florida Blvd., Orlando, FL, 32816
}

\begin{abstract}
Penny Pincher is a recently introduced template matching \$-family gesture recognizer that exhibits competitive accuracy with even just one template. However, our recognizer is also able to rapidly compare a candidate gesture against numerous templates in a short amount of time, as compared to other recognizers, in order to achieve higher accuracy within a given time budget. Penny Pincher achieves this goal by reducing the template matching process to merely addition and multiplication; by avoiding translation, scaling, and rotation; and by avoiding calls to expensive geometric functions. In an evaluation compared against four other \$-family recognizers, in three of our six datasets, Penny Pincher achieves the highest accuracy of all recognizers reaching 97.5\%, 99.8\%, and 99.9\% user independent recognition accuracy, while remaining competitive with the three remaining datasets. Further, when a time constraint is imposed, our recognizer always exhibits the highest accuracy, realizing a reduction in recognition error of between $83 \%$ to $99 \%$ in most cases as Penny Pincher is able to process five times as many templates in the same amount of time as its closest competitor. Further, in this extended work, we also evaluate the effectiveness of Penny Pincher in a stressful setting using a video game prototype that makes heavy use of gestures, so that rushed and malformed gesture articulation is more likely. Our evaluation was conducted with a 24 participant between-subject user study of Protractor and Penny Pincher. Training data and in-game data collected during the user study was further used to evaluate several \$-family recognizers. Again we find that our recognizer is on par with or better than the others, reducing the recognition error by as much as $5.8 \%$ to $10.4 \%$ with just a small number of templates per gesture.
\end{abstract}

Keywords: Gesture recognition, \$-family, template matching

\section{Introduction}

As pen- and touch-based interfaces become ubiquitous via ${ }_{3}$ the widespread use of smart phones, pocket computers, and 4 tablet computers, the need for high quality gesture recognition 5 also continues to rise. Enabling technologies such as the iTunes ${ }_{6}$ Store, Google Play, and Windows Store makes it possible for in7 dividual developers to create and easily distribute small to large 8 scale software for these devices, which has attracted numerous 9 professionals as well as casual hobbyists. However, without ex10 pert knowledge in pattern recognition, a developer is unable to ${ }_{11}$ create even the simplest custom gesture-based interface for his 12 or her software. Wobbrock et al. [1] began to address this issue ${ }_{13}$ in their seminal work when they introduced the $\$ 1$ recognizer. ${ }_{14}$ This is a straightforward, highly accessible, and easy to imple15 ment recognizer that spurred a flurry of research known as the ${ }_{16} \$$-family of recognizers $[1,2,3,4,5,6]$, their common theme 17 being simplicity.

The $\$$-family exhibits several benefits, the most obvious 19 of those being their all around straightforwardness. This trait 20 makes them ideal not only for rapid prototyping, but because ${ }_{21}$ they are also generally accurate, they are a good choice for sit22 uations where existing gesture recognition libraries cannot be

\footnotetext{
${ }^{*}$ Corresponding author.

Email address: etaranta@gmail. com (Eugene M. Taranta II)
}

23 used or when licensing constraints make their use prohibitive. ${ }_{24}$ Since they are quick to implement, \$-family recognizers are 25 also ideal for studies in gesture-based user interfaces. Another ${ }_{26}$ argument for their use, provided by Li [5], is that for custom ${ }_{27}$ gestures, users are unwilling to provide more than a small num28 ber of training examples, which makes statistical based meth29 ods like Rubine's [7] linear discriminator impractical. Further, 30 each recognizer is designed to solve a different problem. For ${ }_{31}$ example, \$1 was designed for unistroke gestures, $\$ \mathrm{~N}$ for mul32 tistroke, \$P for memory reduction, Protractor to overcome the зз speed impediments of $\$ 1,1^{\phi}$ for fast recognition of rotation in34 variant gestures, and so on. However, all of these varieties rely 35 on template matching and as such, tend to improve in accuracy ${ }_{36}$ as the number of training templates increase.

37 We recently introduced Penny Pincher [8] to address the ${ }_{38}$ problem of how to create a recognizer that is accurate within 39 a time constraint. For instance, suppose that the recognizer is 40 part of a game. The recognizer then has to share computational ${ }_{41}$ resources with several other components including AI, the ren42 dering engine, animation system, physics simulator, event and 43 message handlers, networking, scripts, and so on [9]. There${ }_{44}$ fore, a software architect may allocate only 50 microseconds 45 to the gesture recognition task when a new stroke is collected, ${ }_{46}$ to ensure that the process is not disruptive to other game tasks. ${ }_{47}$ This constraint poses two issues. First, with such a tight con${ }_{48}$ straint, certain recognizers cannot be used because they are sim${ }_{49}$ ply too slow. Second, this limits the number of templates a rec- 


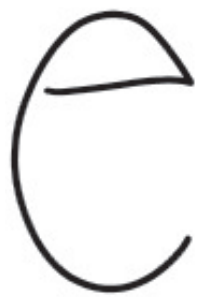

(a) User Specified Gesture

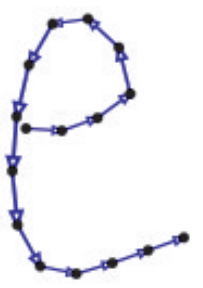

(c) Best Match

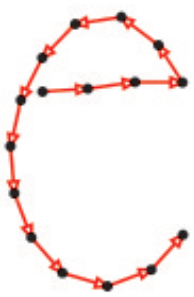

(b) Resample Gesture

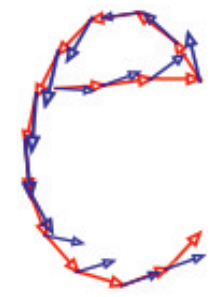

(d) Between-point Vectors Comparison

Figure 1: A candidate gesture (a) is given by the user, which is then resampled into a set of equidistance segments (b). Given a template gesture (c) we compare the corresponding between-point vectors to find the best match. In this case, the 'e' template is the best match because it minimizes the sum of the angles of the corresponding resampled input and template vectors as compared to all other templates.

50 ognizer can check during the matching process. Another con${ }_{51}$ cern is that recognition latency plays a critical role in user per52 ception and interface design. Gray and Boeham-Davis [10], for ${ }_{53}$ instance, have shown that even seemingly negligible latencies ${ }_{54}$ (on the order of milliseconds) can influence how users interact 55 with software. Therefore, to address these issues we introduced ${ }_{56}$ Penny Pincher, an accurate recognizer that achieves high accu57 racy through speed. This $\$$-family extension is designed to do 58 the absolute minimum amount of work possible to match can${ }_{59}$ didate gestures with templates so that more templates can be 60 evaluated within the same amount of time as other recognizers. ${ }_{61}$ As a result, our recognizer is significantly more accurate than 62 its kindred when operating under a time constraint.

63 Most other recognizers use Euclidean distance to compare ${ }_{64}$ gestures whereas we accomplish our goal by comparing between ${ }_{65}$ point vectors, see Figure 1. As do other recognizers, we resam${ }_{66}$ ple the input candidate stroke to a constant number of points; ${ }_{67}$ however, we avoid rotating, scaling, and translating the gesture. ${ }_{68}$ The only limitation of this choice is that our recognizer is not ${ }_{69}$ rotation invariant, but with enough templates loaded, this is not 70 an issue. Further, after resampling the input, we use only ad71 dition and multiplication operations; that is, we also avoid any 72 calls to computationally expensive geometric functions, such as ${ }_{73}$ acos or sqrt.

74 In Section 2 we discuss relevant related work and then we 75 formally present Penny Pincher in Section 3. Although our rec76 ognizer was designed for speed, we found through the detailed ${ }_{77}$ evaluation presented in Section 4 that Penny Pincher also per78 forms on par with other recognizers with only a few templates 79 per gestures. Based on these results, we extended our evalu-

80 ation to consider the accuracy of Penny Pincher and other \$${ }_{81}$ family recognizers in a game environment. Specifically, we de82 veloped the bimodal pen and touch video game Lemarchand's ${ }_{83}$ Prototype to evaluate the effectiveness of such recognizers in ${ }_{84}$ circumstances where gesture articulation is likely to be rushed 85 and often malformed. This is one domain where flexible rec${ }_{86}$ ognizers that can handle a great deal of variance are likely to ${ }_{87}$ be appreciated. In Section 5, we show that Penny Pincher is 88 again on par with or better than other recognizers despite its ${ }_{89}$ simplicity. In Section 6, we discuss and analyze our findings, 90 and future work, and finally we conclude in Section 7.

\section{2. Related Work}

92 Since Rubine's [7] seminal work on gesture recognition, nu93 merous techniques have been developed for both gesture and ${ }_{94}$ symbol recognition. One popular branch of research focuses 95 on easy to understand and easy to implement recognizers. The ${ }_{96} \$$-family of recognizers started with Wobbrock et al. [1] who ${ }_{97}$ designed the $\$ 1$ recognizer. This simple and effective unistroke 98 recognizer works by resampling strokes to a predetermined num99 ber of points, and then these strokes are translated and scaled to 100 the unit square centered at zero. Euclidean distance between 101 points is then used as a similarity metric to compare candidate 102 strokes with template gesture strokes. The template with the 103 least distance is assumed to be the most likely gesture. To en104 sure the best match possible, $\$ 1$ also uses a golden section 105 search (GSS) [11] to rotate candidate strokes into alignment 106 with the template under consideration. Anthony and Wobbrock 107 [2] extended the $\$ 1$ unistroke recognizer into the $\$ N$ multi108 stroke recognizer. This version is able to handle multistroke 109 gestures by combining all strokes into a single stroke so that ${ }_{110} \$ 1$ techniques can be leveraged. Given an example gesture, 111 during training when templates are constructed, all permuta112 tions of strokes and stroke directions are used to generate all 113 possible ways in which the gesture can be written.

114 While the previous approaches work with arcs, Vatavu et al. 115 [6] considers input as point clouds. The \$P recognizer still re${ }_{116}$ samples, scales, and translates strokes and uses Euclidean dis117 tances as a similarity metric. However, the points are not treated 118 as a time series; instead the authors employ a greedy algo119 rithm that tests different permutations of points to find the over120 all minimum distance between candidate and template point ${ }_{121}$ clouds. Herold and Stahovich [4], on the other hand, continue 122 to work with strokes as time series, but their $1^{\phi}$ recognizer gen${ }_{123}$ erates a rotation invariant representation that is a time series 124 vector of one-dimensional points-normalized distances mea125 sured from the stroke's centroid. That is, strokes are resam126 pled as usual, but then each two-dimensional point is converted 127 into a one-dimensional scalar, the distance of the point from the 128 centroid normalized by the standard deviation of all distances. ${ }_{129}$ Candidate and template strokes are then compared using the 130 one-dimensional Euclidean distance.

131 One major limitation of $\$ 1, \$ N$, and $\$ P$ is that they are gen${ }_{132}$ erally slow. The former two recognizers are slow due to their 133 use of the GSS. Li [5] was able to overcome this limitation. He ${ }_{134}$ discovered a closed form equation to find the optimal rotation 
135 of a candidate stroke to match the template. Since Li's Protrac136 tor recognizer represents each stroke as a vector and works with ${ }_{137}$ the angle between the two vectors (between the candidate and 138 template vectors), it is not necessary to scale candidate strokes 139 when matching. However, resampling and translation to the 140 centroid are still required. Anthony and Wobbrock [3] adapted ${ }_{141}$ Protractor's technique to create a fast multistroke recognizer, 142 \$N-Protractor.

${ }_{143}$ To further speed up template matching, Reaver et al. [12] ${ }_{144}$ proposed Quick\$ to use agglomerative hierarchical clustering 145 with a branch and bound search to cull branches that cannot ${ }_{146}$ contain a better match than what has already been found. Zhang 147 et al. [13] use nonlinear embedding [14, 15] as a filter to quickly 148 check the lower boundary of the Euclidean distance between a 149 candidate and template using only stroke means and variances 150 based on a low dimensional representation. Vatavu [16] de${ }_{151}$ veloped $1 \mathrm{~F}$ to sort all templates based on a scalar value (such 152 as total curvature). Then the probability mass functions ( $\mathrm{pm} f$ ) ${ }_{153}$ are learned for each template. During the matching process, 154 the best match is identified based on the $1 \mathrm{~F}$ value, and then the ${ }_{155} \mathrm{pm} f$ of the associated template is used to determine how far left 156 and right of the current template to search for the best match. ${ }_{157}$ Except for nonlinear embedding, our Penny Pincher recognizer 158 is orthogonal to these methods. However, such methods are 159 not within the spirit of the \$-family because of their additional 160 complexity in code and data structures.

161 Fuccella and Costagliola [17] more recently introduced a 162 new recognizer that the authors claim is also suitable for rapid ${ }_{163}$ prototyping, and that outperforms other state-of-the art recog164 nizers. However, their recognizer is somewhat more complex 165 than the typical \$-family recognizer, involving polyline approx166 imation using dominant points, smoothing of polylines by seg167 ment fusion, polyline segment alignment, rotation with an op168 tional GSS, and a final distance calculation.

169 Compared to other \$-family recognizers, Penny Pincher [8] 170 is the easiest yet to understand and simplest to implement. As 171 mentioned previously, we do not rotate, scale, or translate can172 didate gestures, whereas every other recognizer does at least 173 one of these. Also, most recognizers use Euclidean distance 174 and have to make calls to geometric functions at some point 175 in the template matching process. We compare between-point 176 vectors instead, so that only simple dot product calculations are 177 required (after the gesture has been resampled). As we show in ${ }_{178}$ Section 4, these simple modifications improve computational 179 performance without sacrificing accuracy. However, the main 180 contribution of this paper is an extension of our previous evalu181 ation, where Penny Pincher and other \$-family recognizers are 182 put to test in a challenging video game environment. Again, 2 183 we demonstrate that our recognizer is ideal for its accuracy 184 and simplicity, as compared to the alternatives, in this tough 185 domain-one in which players are rushed, make mistakes, and 186 write sloppy.

\section{3. Penny Pincher}

188 Loosely following [16], we define a gesture as an ordered ${ }_{189}$ series of points as follows:

$$
g=\left\{g_{i}=\left(x_{i}, y_{i}\right) \in \mathbb{R}^{2}\right\},
$$

190 where $i$ indexes the gesture's points in time relative order. Ges191 tures are resampled by length into $n$ points so that the distance 192 between each point is equal. A gesture is then converted into a 193 series of between-point vectors:

$$
v=\left\{v_{i}=g_{i+1}-g_{i}\right\},
$$

194 for $i<n$. Given two gestures $v$ and $w$, their similarity is taken to 195 be the sum of the angles between corresponding between-point 196 vectors:

$$
D(v, w)=\sum_{i=1}^{n-1} \frac{v_{i} \cdot w_{i}}{\left\|v_{i}\right\|\left\|w_{i}\right\|} .
$$

${ }_{197}$ A perfect score is therefore $n-1$ because the normalized dot 198 product is 1 for identical vectors. Now, let $m$ be the number of 199 distinct gestures and $\mathcal{T}$ the set of templates created by training 200 the recognizer:

$$
\mathcal{T}=\left\{t_{i}=\left(s_{i}, l_{i}\right) \mid l_{i} \in\{1 \ldots m\}\right\},
$$

201 where $t_{i}$ is the enumerate template in set $\mathcal{T}, s_{i}$ is the gesture 202 sample's between-point vector, and $l_{i}$ is the gesture's class label. ${ }_{203}$ A candidate gesture $c$ takes the classification of the template $T$ 204 that is most similar:

$$
T=\underset{t_{i} \in \mathcal{T}}{\arg \max } D\left(t_{i}, c\right),
$$

205 This already simple calculation can be simplified further. ${ }_{206}$ During training, let the components of the between-point vec207 tors be normalized so that each $\left\|s_{i}\right\|=1$, thus eliminating one 208 normalization factor in Equation 3. Next, as a simplifying as209 sumption, say that the length of each between-point vector com210 ponent is equal (each $\left.\left\|w_{i}\right\|=\left\|w_{i+1}\right\|\right)$ because gestures are re211 sampled into $n-1$ equidistance arc lengths; this assumption 212 is evaluated in Section 4.2. This allows us to treat $\left\|c_{i}\right\|^{-1}$ as 213 a constant to be factored out of the summation of Equation 3. ${ }_{214}$ Further, note that this factor is the same for all evaluated tem${ }_{215}$ plates in Equation 5-it scales each dot product result identi${ }_{216}$ cally. Therefore the scaling factor can be eliminated entirely 217 without affecting the final result, thus simplifying our template 218 matching equation to:

$$
T=\underset{t_{i} \in \mathcal{T}}{\arg \max } \sum_{i=1}^{n-1} v_{i} \cdot w_{i} .
$$

There are a few distinct advantages to this formulation. No220 tice first that there are no rotation, scale, or translation opera221 tions involved. This means less overhead in preparing templates 222 and matching. Also, template matching is reduced to merely ${ }_{223}$ addition and multiplication with no expensive calls to geomet${ }_{224}$ ric library routines. This reduction in overhead compared to 225 other recognizers means that more templates can be evaluated 226 in the same amount of time, see Section 4. The simplicity of our 227 approach also means Penny Pincher is the easiest to understand 228 and implement in the \$-family. 
229 It should be noted that Penny Pincher is not specifically a 230 multistroke gesture recognizer. However, we leverage the same ${ }_{231}$ technique used by $\$ \mathrm{~N}$ [2], which is to concatenate sequential ${ }_{232}$ strokes together into a single unistroke gesture. We find that 233 with a sufficient number of training samples, this achieves the 234 same effect as \$N's scheme to internally generate all possible 235 permutations of a gesture from a single sample.

\section{3.1. Complexity}

${ }_{237}$ In this section we discuss the theoretical minimum asymp238 totic boundary of the $\$$-family of recognizers. Without the use ${ }_{239}$ of filtering or other culling techniques we make the following 240 assumptions:

1. Candidate gestures are always resampled.

2. The resampled candidate gesture is always compared against every template in $\mathcal{T}$.

3. Each point in the resampled candidate gesture is evaluated against at least one corresponding template point.

${ }_{246}$ Under these assumptions, let $r=|c|$ be the length of the can247 didate gesture before resampling, representing the number of ${ }_{248}$ raw data points. As before, $n$ is the number of resampled data 249 points. The cost of resampling $c$ is the cost of first calculat250 ing the stroke length and then computing the resample points. ${ }_{251}$ This requires touching every raw data point twice, $\Omega(2 r)$, and ${ }_{252}$ every resample point once, $\Omega(n)$. The latter is because each ${ }_{253}$ resample point is part of the path and must be considered as 254 the resampling procedure continues. Supposing that the rec255 ognizer can match the resampled points directly (e.g., without 256 further manipulation), then no additional processing is neces257 sary. Template matching is subsequently carried out for each ${ }_{258} t \in \mathcal{T}$, which is $\Omega(n|\mathcal{T}|)$. Therefore, at best, a template based 259 recognizer as described is bounded by:

$$
\Omega(2 r+n+n|\mathcal{T}|) .
$$

260 To the best of our knowledge, Penny Pincher is the first ${ }_{261} \$$-family recognizer to achieve this lower bound. Note that ${ }_{262}$ the complexity of several other recognizers are available in [6], ${ }_{263}$ however, these do not consider the cost of resampling. Of course ${ }_{264} \Omega$ notation hides certain details that are important in practice. ${ }_{265}$ We have to consider that $1^{\phi}$, for example, represents each point 266 in its template as a one-dimensional point where the other meth${ }_{267}$ ods use two-dimensional points. This means that $1^{\phi}$ can com268 pare templates faster despite having a non optimal resampling 269 strategy as is shown in the evaluation Section 4.3. Penny Pincher, 270 on the other hand, relies on straightforward dot product calcula271 tions without having to utilize external or built-in math libraries.

\section{4. Evaluation}

273 We evaluate Penny Pincher using three different tests. All 274 tests were performed on a MacBook Pro with a $2.4 \mathrm{GHz}$ Intel ${ }_{275}$ Core i7 processor, $8 \mathrm{~GB}$ of $1333 \mathrm{MHz}$ DDR3 memory, and an 276760 GB mechanical SATA disk. In our first test we check the 277 validity of the assumption that all between-point vector lengths
278 of a single gesture are of equal length. Next we evaluate the 279 accuracy of our method compared to other \$-family recogniz280 ers when the number of templates is controlled (the standard ${ }_{281}$ method of evaluation). Finally we investigate the accuracy of 282 the fastest recognizers to see how well they perform under vary283 ing time constraints. However, we first describe the various ${ }_{284}$ datasets used in our tests in the follow subsections.

\section{4.1. Datasets}

This subsection gives a brief description of each dataset 287 used in our evaluation. For additional information beyond what 288 is presented here, we refer the reader to the associated cited 289 Work.

290 \$1-GDS. The \$1 recognizer gesture dataset [1] is a unistroke 291 gesture dataset comprising 16 gestures collected from 10 substs jects at 3 speeds (slow, medium, and fast) on an HP iPAQ h4334 293 Pocket PC. Because each subject supplied 10 samples of each ${ }_{294}$ gesture at each speed, there are 4800 samples in aggregate.

${ }_{295}$ SIGN. The Synchromedia-Imadoc Gesture New On-Line 296 Database [18] is a unistroke gesture dataset comprising 17 classes 297 collected from 20 writers on tablet PCs and whiteboards. Each 298 writer supplied 25 samples over four sessions so that SIGN con299 tains 8500 samples in aggregate.

EDS 1 and EDS 2. Two unistroke gesture datasets were col301 lected by Vatavu et al. [19] to study gesture execution difficulty, 302 referred to as Execution Difficulty Set \#1 (EDS 1) and Execu303 tion Difficulty Set \#2 (EDS 2). The first dataset contains 5040 304 samples in aggregate comprising 18 gestures collected from 14 305 participants providing 20 samples each. The latter dataset con306 tains 4400 samples in aggregate comprising 20 gestures from 30711 participants providing 20 samples each. All samples were 308 collected on a Wacom DTU-710 Interactive Display.

309 MMG. The Mixed Multistroke Gestures dataset [3] com310 prises 16 gestures having between one and three strokes. Sam${ }_{311}$ ples were collected from 20 participants using either a stylus 312 or their finger on a tablet PC at three different speeds (slow, 313 medium, and fast). In aggregate there are 9600 samples.

UJI. The UJIpenchars2 Database [20] is a multistroke ges315 ture dataset of lowercase and uppercase letters, digits, charac316 ters with diacritics, and punctuation marks containing an aggre317 gate of 11640 samples over 97 gesture classes. Data was col318 lected from 60 writers each providing 2 samples of each class.

\section{4.2. Verification of Between-Point Vector Distribution}

The simplifying assumption used in Equation 6 supposes ${ }_{321}$ that each between-point vector is of equal length. In reality, 322 this is inaccurate because indeed, vectors taken from collinear 323 points are longer than those taken from curves, which in turn ${ }_{324}$ are generally longer than those taken from sharp cusps. How325 ever, for the purpose of an approximation, we find this assump326 tion to be sufficient. To verify this we examine the empiri327 cal probability density distribution generated with the relative 328 between-point vector lengths of each sample in six datasets (\$1${ }_{329}$ GDS, SIGN, EDS 1, EDS 2, MMG, and UJI). For each individззо ual sample, the mean between-point vector length is computed ${ }_{331}$ and all vectors within the same sample are normalized by the 


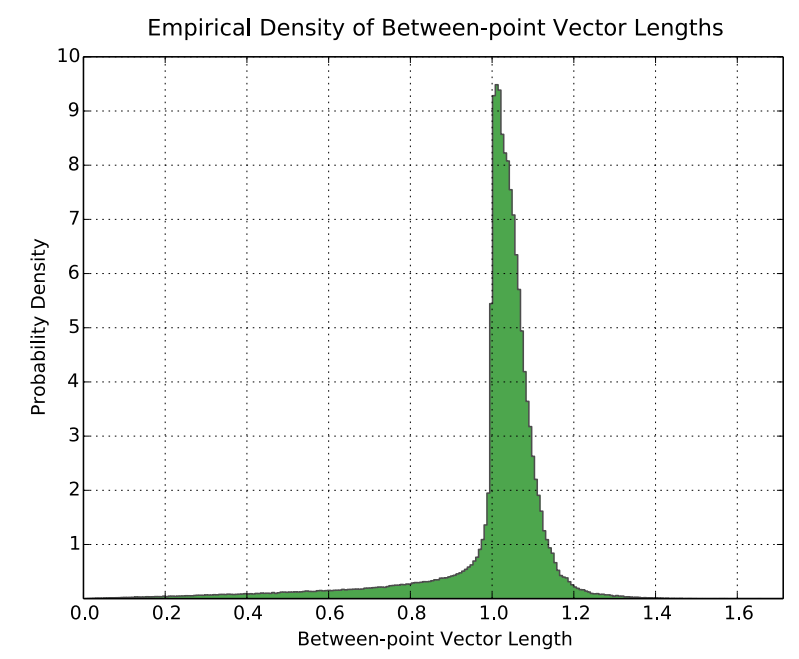

Figure 2: The empirical probability density of the distribution of between-point vector length. For each resampled gesture in the \$1-GDS, SIGN, EDS 1, and EDS 2 datasets, we normalize the between-point vectors to the average length of the vectors within the gesture. All results are combined to create this overall distribution of relative lengths. It can be seen that the majority of the density is centered around 1.0 which indicates that the majority of between-point vectors are of equal length within each sample.

332 mean. Then the set of all normalized vectors from all sets are ${ }_{33}$ combined to build the empirical probability density distribution ${ }_{334}$ shown in Figure 2. If all between-point vectors were truly of 335 equal length then the full density would be located at one. We 336 see, though, that the majority of the density is near one with 337 the distribution being left skewed. Specifically, $85 \%$ of the dis338 tribution is contained between 0.9 and 1.15 and is left skewed $339-2.85$. The individual distributions of the six unique datasets ${ }_{340}$ are all similar to the overall distribution. They are left skewed 341 in the same manner $(-3.08,-3.06,-2.44,-2.56,-2.5$, and -2.59 342 respectively) and contain a similar portion of the density within ${ }_{343}$ the same range $(87.58 \%, 87.43 \%, 79.96 \%, 85.83 \%, 78.81 \%$ 344 and $81.06 \%$ respectively). Further, note that the observed left 345 skewness is expected. When most vectors are of similar length 346 but there are a small number of short vectors on sharp cusps 347 for example, then the mean is pulled down, shifting the dens348 est portion of the distribution right of the mean. Nevertheless, ${ }_{349}$ given that the majority of the density is located near one, we 350 find our previous assumption that between-point vector lengths 351 are approximately equal to be accurate enough for our use.

\section{4.3. Standard Test}

${ }_{353}$ Using all six datasets we compared the accuracy of Penny ${ }_{354}$ Pincher against its predecessors \$1, Protractor, \$N, \$N-Protractor 355 and $1^{\phi}$ using what we call the standard test. The resampling 356 rate of each recognizer was set according to that given (or sug357 gested) in each recognizer's paper respectively: $1^{\phi}$, Protrac358 tor, and Penny Pincher resample to 16 points whereas $\$ \mathrm{~N}, \$ \mathrm{~N}$ ${ }_{359}$ Protractor, and $\$ 1$ resample to 96 points. Where applicable, we 360 enabled bounded rotation invariance due to an observed slightly ${ }_{361}$ higher recognition performance. Note that we also considered ${ }_{362}$ \$P. However, we found that the recognizer was very slow in
363 practice, and based on results reported in [6], this recognizer ${ }_{364}$ performs similarly to $\$ 1$ for unistroke gestures. Also because 365 we are interested in recognizers that can potentially process nu366 merous templates in a short amount of time, \$P was not an op367 tion.

${ }_{368}$ In this part of the evaluation, the template count $T$ varies 369 from 1 to 10 for each gesture and we consider only user inde370 pendent scenarios. For each template count and for each recog371 nizer we ran 1000 tests. That is, in each iteration we randomly 372 select $T$ templates per gesture from all of the available samples, 373 and then we randomly select one remaining sample from each 374 gesture to be the candidate gesture. Therefore each test con375 tains $g$ recognition tasks, where $g$ is the number of gestures in 376 the dataset. Throughout the following, we report on the recog377 nition error of the various recognizers (which is equivalent to 378 one minus the accuracy). All of the standard test results can be 379 found in Figure 3.

Penny Pincher performs strongly on the \$1-GDS dataset. ${ }_{381}$ With only one template loaded, the error rate is $8.58 \%$ which 382 drops to $1.3 \%$ by ten templates. $\$ 1$, on the other hand, also 383 starts at $8.58 \%$ but only achieves a $1.9 \%$ error by the end. Pro384 tractor makes the most dramatic improvement in reduction of 385 error by swinging from $12.16 \%$ to $1.55 \%$, beating out $\$ 1$ start${ }_{386}$ ing at 6 templates. For the EDS 1 dataset, Penny Pincher is 387 mostly a middle of the road yet still accurate performer, achiev38 ing a $3.3 \%$ error with one template, and dropping to below $0.7 \%$ with three templates, but reaching the best result of all recog390 nizers at $0.11 \%$ error with ten templates loaded. With EDS 2, 391 our recognizer starts at $2.19 \%$ and drops to $.70 \%$ by the second 392 template. For the remaining template counts, Penny Pincher 393 maintains the highest accuracy with its best result at 9 templates 394 ( $0.11 \%$ error). Both $\$ 1$ and Penny Pincher perform very well 395 compared to $\$ \mathrm{~N}$ and Protractor for the SIGN dataset, although ${ }_{396}$ Penny Pincher has better overall performance. We start at a $39713.41 \%$ error which drops over ten templates to $2.2 \%$, whereas ${ }_{398} \$ 1$ starts at $13.47 \%$ and only drops to $4.41 \%$

$399 \quad \mathrm{MMG}$ is a dataset designed for $\$ \mathrm{~N}$ and $\$ \mathrm{~N}$-Protractor which 400 were developed to handle multistroke gestures. So it is expected 401 that Penny Pincher would not perform as well in this case. Our 402 recognizer is approximately in line with Protractor, where with 403 one template loaded, we see $38.31 \%$ error. This steadily de404 creases until the error rate reaches $8.0 \%$ using 10 templates. 405 One thing to note, however, is that $\$ \mathrm{~N}$ internally creates a tem406 plate for every possible permutation of the strokes and their di407 rections. So although the template count is 10 , for example, 408 in reality $\$ \mathrm{~N}$ is evaluating significantly more templates. As is 409 shown in the third part of our evaluation, Penny Pincher is also 410 capable of achieving similar or better results when more tem${ }_{411}$ plates are available. UJI is a large gesture dataset that has ges${ }_{412}$ tures with significant similarities, making it a difficult dataset ${ }_{413}$ for all recognizers. Our recognizer starts out at a $67.12 \%$ error 414 rate and only achieves $41.53 \%$ error with ten templates loaded. 415 This is approximately in line with with $\$ 1$ and Protractor. \$N, ${ }_{416}$ however, fares less well, only reaching $45.32 \%$

In all of the six datasets, $1^{\phi}$ 's accuracy was well below the ${ }_{418}$ other recognizers. This is likely due to the fact that $1^{\phi}$ is com${ }_{419}$ pletely rotation invariant, and while this turns out to be good 

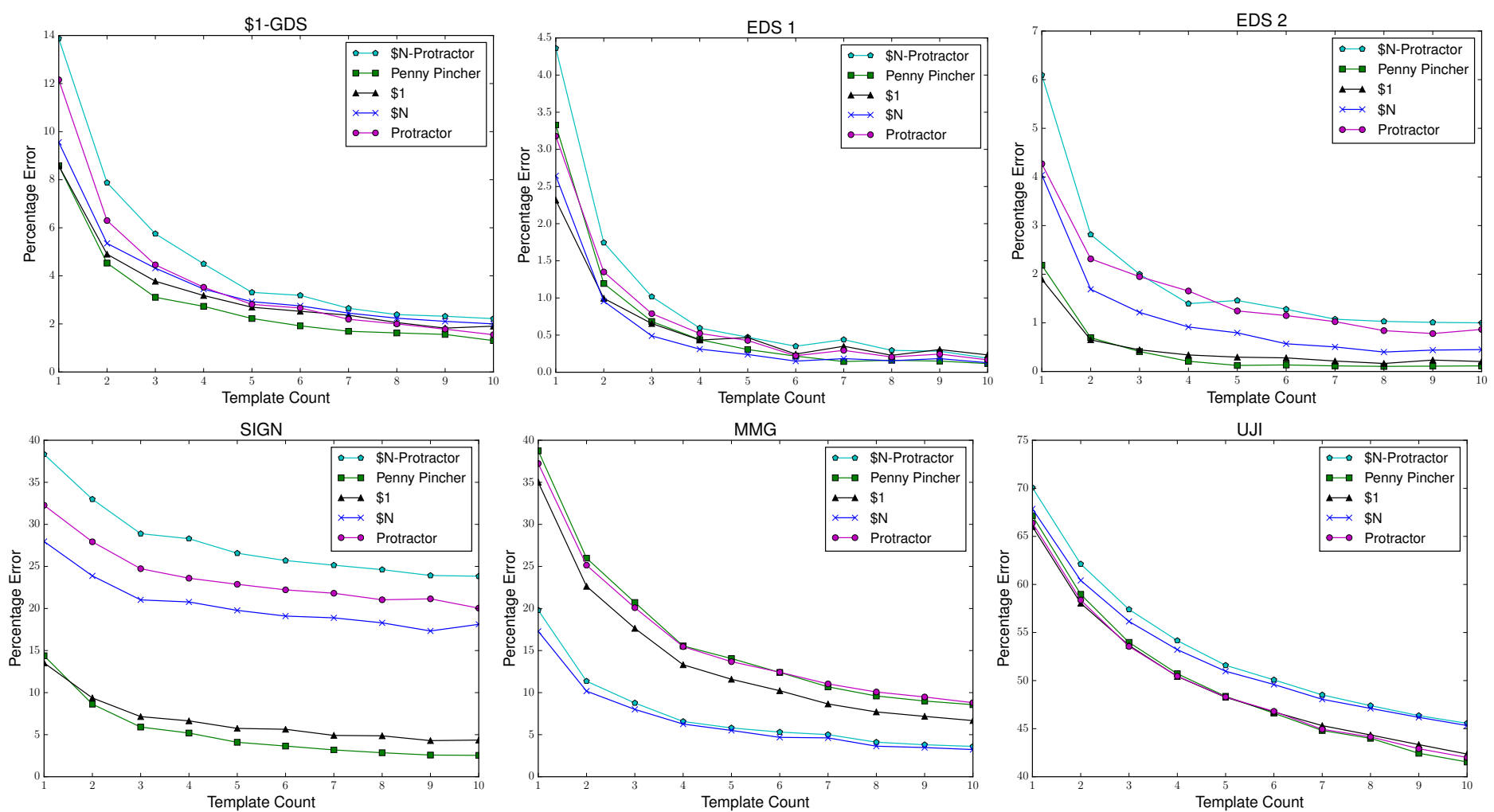

Figure 3: User independent error recognition test results for varying template counts. Each test, for each recognizer and template count, was performed 1000 times. $1^{\phi}$ was also tested, though because it exhibited high error rates with these datasets, it was removed for readability.

\begin{tabular}{lcc}
\hline Recognizer & Avg ns / Template & ns- $\sigma$ \\
\hline $1^{\phi}$ & 25 & 4.5 \\
\hline Penny Pincher & 33 & 6.2 \\
\hline Protractor & 174 & 27.7 \\
\hline \$N-Protractor & 2449 & 650 \\
\hline
\end{tabular}

${ }_{436}$ one reason why Penny Pincher is able to achieve high accuracy 437 as reported in the next subsection.

\section{${ }_{438}$ 4.4. Budget Test}

${ }_{439}$ We refer to our final test as the budget test. In this scenario 440 we are given a time constraint and are allowed to process as

Table 1: Average time taken in nanoseconds per template to perform the recognition task for the UJI dataset given 10 templates per gesture. The four fastest recognizers are shown alongside their standard deviations. Although $1^{\mathscr{f}}$ is the fastest recognizer, its accuracy is subpar compared to its competitors for this dataset.

420 for computational performance, the effect is that it is not a good ${ }_{421}$ general purpose recognizer. Table 1 shows computational per422 formance of the fastest recognizers. This was determined by ${ }_{423}$ averaging together the duration of each of the individual tests 424 for the UJI dataset for ten templates, which was then divided by 425 the total number of loaded templates $(T * g)$. The result is the 426 time it takes to compare one candidate gesture to one template ${ }_{427}$ gesture (though note that the cost of resampling the candidate ${ }_{428}$ gesture is amortized over all comparisons). $1^{d}$ is the fastest 429 recognizer being able to compare two gestures in 25 nanosec430 onds. Penny Pincher is also vey fast, achieving a 33 nanosec${ }_{431}$ ond benchmark. Though $1^{\phi}$ is faster, its accuracy prohibits its ${ }_{432}$ general use as we saw when working with the six test datasets. ${ }_{433}$ Finally, Protractor, relative to the top two recognizers, is slow ${ }_{434}$ in comparison, taking 174 nanoseconds to complete one check, ${ }_{435}$ which is 5.27 times Penny Pincher. This difference in speed is

441 many templates as possible within the given boundary. This 442 setup is similar to the standard test except that we vary the time ${ }_{443}$ constraint rather than the template count directly. For each test 444 we first determine the number of templates the recognizer can 445 process per second. We then start with a $10 \mu$ s budget and al446 low the recognizer to train with as many templates as it can 447 process within the time constraint. Using this configuration, as 448 before, we execute 1000 randomized tests. Thereafter the bud${ }_{449}$ get is incremented by $10 \mu \mathrm{s}$ and the test is rerun. This continues 450 until there are not enough remaining samples to perform a full ${ }_{451}$ recognition test. Since the number of templates a recognizer 452 can train will depend on the budget, it is possible to have an 453 uneven number of templates per gesture class. If, for example, 454 the budget allows for 2.2 templates per gesture, we ensure that 455 at least two templates are loaded per class and then a random ${ }_{456} 20 \%$ of the gesture classes will have a third template loaded. ${ }_{457}$ The results of this test are shown in Figure 4. The graphs are 458 cropped to $100 \mu$ s for readability, but the tail results are reported 459 below.

Penny Pincher achieves high accuracy for EDS 2 right at the ${ }_{461}$ start with a $0.13 \%$ error and is able to process approximately 7.9 462 templates per gesture (tpg) within $11 \mu \mathrm{s}$. As the test continues, 

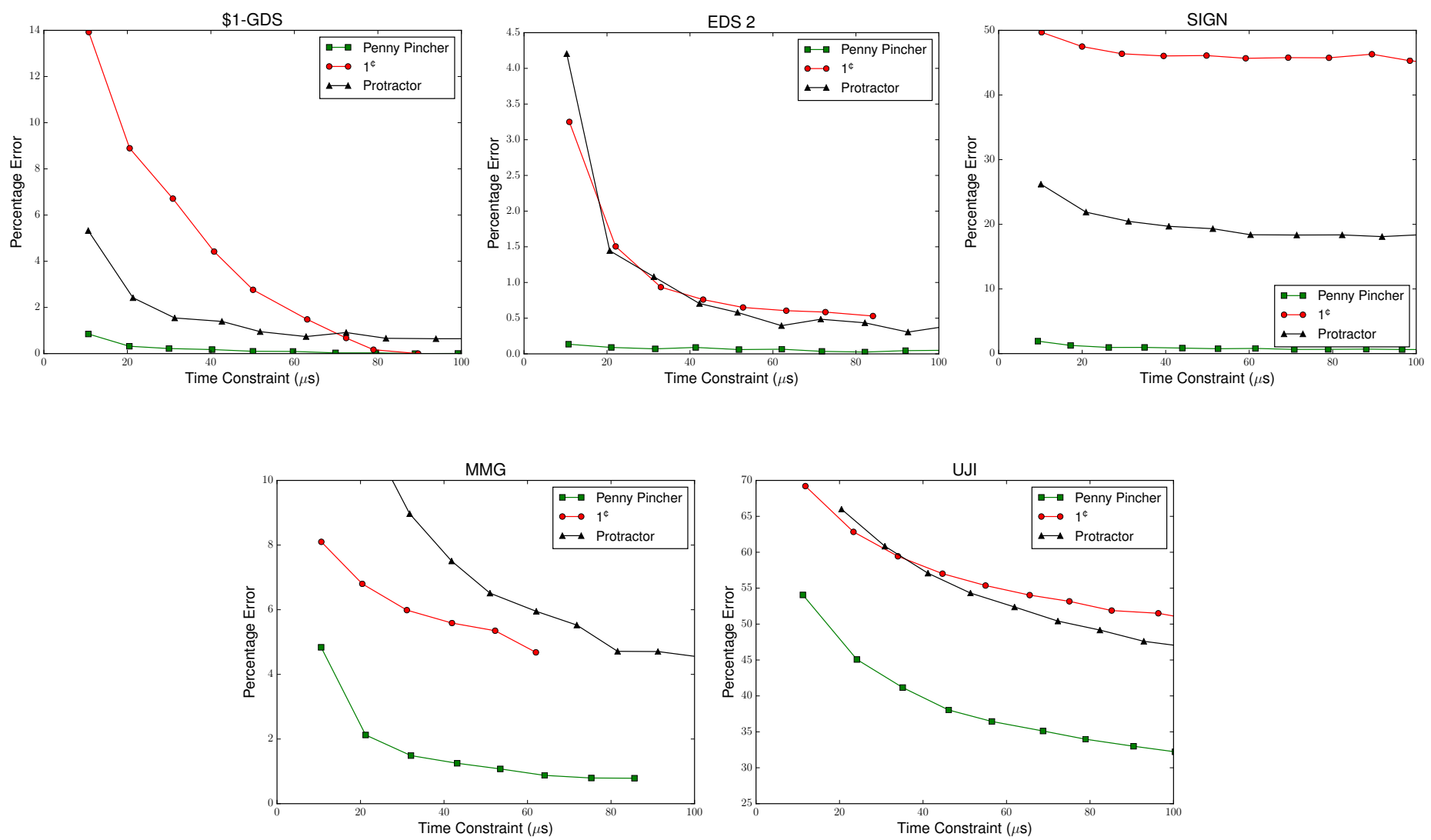

Figure 4: User independent error recognition test results for varying time constraints. Each test, for each recognizer and budget, was performed 1000 times. Although the graphs are cropped to $100 \mu$ s for readability, the tail results are reported in Section 4.4.

\begin{tabular}{lcccccccccc}
\hline & $\mathbf{2 0} \mu \mathbf{s}$ & & $\mathbf{4 0} \mu \mathbf{s}$ & & $\mathbf{6 0} \mu \mathbf{s}$ & & $\mathbf{8 0} \mu \mathbf{s}$ & & $\mathbf{1 0 0} \mu \mathbf{s}$ \\
\hline \$1-GDS & $87 \%$ & $(0.32,2.41)$ & $87 \%$ & $(0.18,1.39)$ & $87 \%$ & $(0.09,0.74)$ & $95 \%$ & $(0.03,0.66)$ & $99 \%$ & $(0.01,0.64)$ \\
\hline EDS 2 & $94 \%$ & $(0.09,1.45)$ & $87 \%$ & $(0.09,0.71)$ & $84 \%$ & $(0.07,0.40)$ & $94 \%$ & $(0.03,0.44)$ & $87 \%$ & $(0.05,0.38)$ \\
\hline SIGN & $94 \%$ & $(1.26,21.88)$ & $95 \%$ & $(0.96,19.69)$ & $96 \%$ & $(0.75,18.38)$ & $96 \%$ & $(0.65,18.36)$ & $96 \%$ & $(0.69,18.40)$ \\
\hline MMG & $69 \%$ & $(2.13,6.80)$ & $83 \%$ & $(1.25,7.51)$ & $85 \%$ & $(0.88,5.95)$ & $83 \%$ & $(0.79,4.71)$ & - & - \\
\hline UJI & $26 \%$ & $(45.07,60.84)$ & $30 \%$ & $(38.04,54.32)$ & $30 \%$ & $(35.12,50.42)$ & $31 \%$ & $(32.99,47.60)$ & $31 \%$ & $(31.41,45.7)$ \\
\hline
\end{tabular}

Table 2: Reduction in percentage error for 20, 40, 60, 80 and 100 microsecond budgets. Shaded cells show the percentage reduction in error achieved by Penny Pincher compared to the second most accurate recognizer at each sample location (see the individual graphs in Figure 4 to know which recognizer this is). To the right of each percentage reduction result is the exact percentage error of Penny Pincher and its competitor, in this order. Note that with MMG, the dataset is exhausted before Penny Pincher reachers $100 \mu$ s, which is why there is no entry.

${ }_{463}$ the dataset is exhausted at a $111.29 \mu$ s budget where the error ${ }_{464}$ rate drops to $0.06 \%$ and 208.6 tpg are processed. $1^{\phi}$ initially 465 achieves a $3.2 \%$ error with 10.4 tpg at a $11 \mu$ s budget. This ${ }_{466}$ improves to only $0.53 \%$. Protractor starts with the worst accu$467 \mathrm{racy}$, only being able to handle $1.05 \mathrm{tpg}$ at $10 \mu \mathrm{s}$. As expected, ${ }_{468}$ Protractor takes the longest to complete where the dataset is ex${ }_{469}$ hausted at $701.34 \mu \mathrm{s}$, though the error rate drops to $0.1 \%$. In 470 all budgets, Penny Pincher achieves a considerably lower error 471 rate.

472 Of all our tests, $1^{\phi}$ achieves its best results with the $\$ 1$ ${ }_{473}$ GDS budget test. Although the initial error is high (14\% at $47419.12 \mathrm{tpg}$ ), this drops to $0 \%$ by the end with 297.94 tpg at $90 \mu \mathrm{s}$. 475 Penny Pincher also reaches $0 \%$ with 219.38 tpg at $89 \mu$ s. How476 ever, its initial error is much better, $0.85 \%$ with $19.12 \mathrm{tpg}$ at $47711 \mu \mathrm{s}$. Starting with $2.81 \mathrm{tpg}$, Protractor only achieves a $5.3 \%$ 478 error and at its best, cannot reach $0 \%$. Using 297.56 tpg over
${ }_{479} 766 \mu \mathrm{s}$, Protractor reaches $.2 \%$ error.

UJI again is the most challenging dataset. Even at 65.81 ${ }_{481}$ tpg and $135 \mu \mathrm{s}$, the error only drops to $49 \%$ from $69 \%$ for $1^{\phi}$. ${ }_{482}$ Protractor is a little better, getting down to $30 \%$ from $66 \%$ but ${ }_{483}$ also requiring a $1152 \mu$ s budget to do so. Penny Pincher has the 484 best performance overall. With 3.06 tpg at $11 \mu \mathrm{s}$, the initial error 485 is $54 \%$, which steadily drops to $28 \%$ by $183 \mu \mathrm{s}$.

$486 \quad$ Initially Penny Pincher did not do as well as \$N when work${ }_{487}$ ing with the MMG multistroke dataset. However, as discussed, ${ }_{488} \$ \mathrm{~N}$ internally creates the various permutations of a gesture from 489 a single template, which implies that given a specific template 490 count, the recognizer is actually processing significantly more 491 variations. In this scenario, that is no longer true. Each recog492 nizer is trained with numerous examples as afforded by the bud493 get so that most practical variations of the gestures are learned 494 by the recognizer (whereas $\$ \mathrm{~N}$ may generate permutations that 


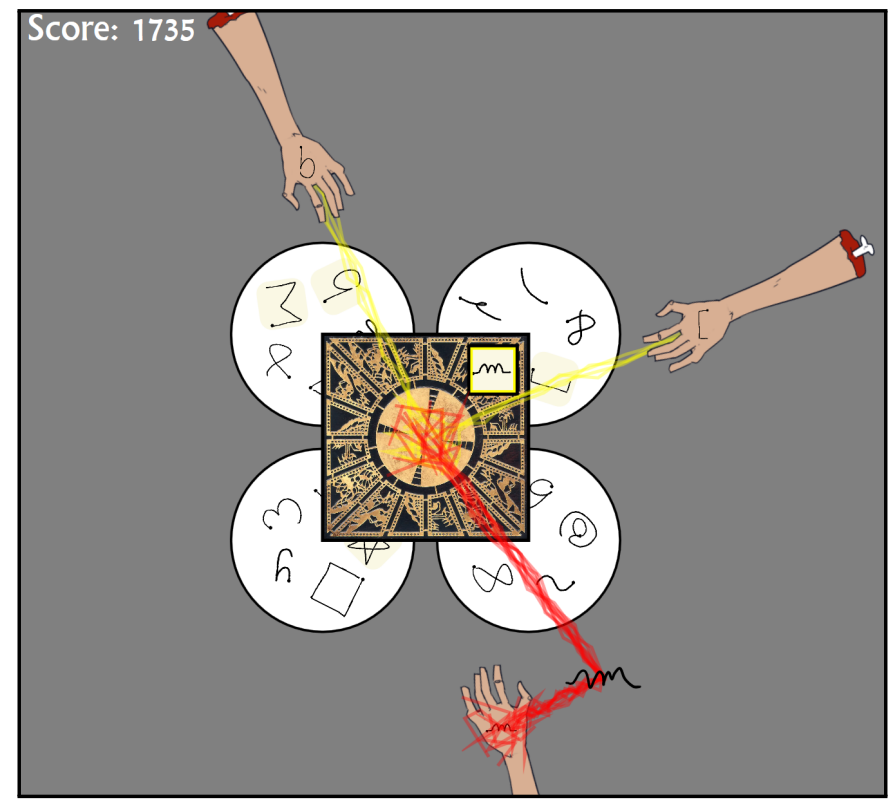

Figure 5: Screenshot of Lemarchand's Prototype, a game designed for penbased gesture input. The box and wheels are rotated with touch. If the symbol in the box's window matches the symbol on the hand of an enemy, then the player can draw the symbol to banish it. Enemies move faster and are generated faster as the game progresses.

495 never occur in practice). Therefore Penny Pincher working 496 with $18.75 \mathrm{tpg}$ at $11 \mu \mathrm{s}$ can achieve a $4.8 \%$ error, which de497 clines rapidly to $1.1 \%$ (42.19 tpg) at $21 \mu \mathrm{s}$, and then to $0.79 \%$ at $49886 \mu \mathrm{s}$ (183.06 tpg). Protractor and $1^{\phi}$ also see higher accuracies 499 but do not fare as well as Penny Pincher. Respectively their best 500 errors rates are $2.9 \%$ and $4.7 \%$ (198 tpg at 526 $\mu$ s and 187 tpg at $50162 \mu \mathrm{s})$.

502 SIGN again is where we see the most dramatic difference 503 in performance between Penny Pincher and the other recogniz504 ers. With a budget of $9 \mu \mathrm{s}$ and 14.29 tpg, the error is already $5051.9 \%$. This continues to improve until the end where the error 506 drops to $0.54 \%$ (with 1936 tpg over $1213 \mu \mathrm{s}$ ). $1^{\phi}$ struggles ini507 tially in comparison with a $50 \%$ error that only drops to $43 \%$ 508 near the end with $747 \mu \mathrm{s}$. Protractor is in the middle with an 509 initial $26 \%$ error (2.41 tpg) which improves only to $14 \%$ with a $5105720 \mu$ s budget.

511 In Table 2 we provide a summary of select results from the 512 budget test. For each sample, we compare Penny Pincher to the 513 second best performing recognizer in the category and report 514 the percentage reduction in recognition error. The exact errors 515 are shown next to each result. It can be seen that in all cases, ${ }_{516}$ Penny Pincher significantly outperforms the other recognizers. ${ }_{517}$ With four datasets, the reduction in recognition error ranges be518 tween $69 \%$ and $99 \%$ with most results being above $83 \%$. Our 519 worst result is observed with the UJI dataset where the recogni520 tion error is only improved by $26-31 \%$.

\section{${ }_{521}$ 5. In Game Evaluation}

522 There are a number of practical applications for pen and 523 touch gesture recognition, though one of particular interest to

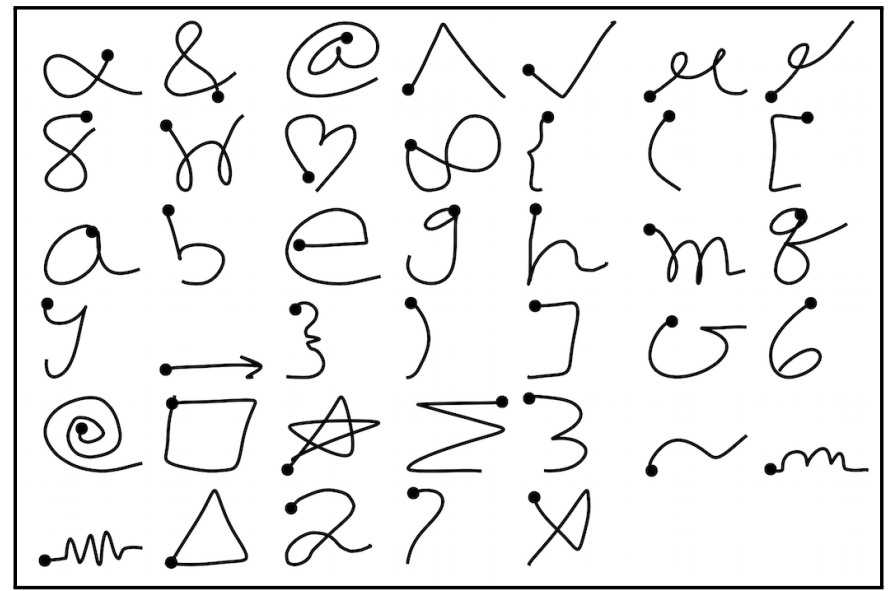

Figure 6: The 40 gestures used in Lemarchand's Prototype. These are randomly selected samples collected from participants during training.

524 us is in the electronic games domain. There are two reasons 525 for this: use cases are limited only to the imagination of game 526 designers, and gesture recognition in games is difficult, often 527 falling below expectation. Baseline results from offline tests, ${ }_{528}$ for example, are often better than those results collected in a 529 video game environment $[21,22]$, which in part may be due to 530 the induced stress associated with playing a game itself. There${ }_{531}$ fore, to evaluate the effectiveness of Penny Pincher and other \$532 family recognizers in this difficult but practical situation, we de533 veloped the toy game Lemarchand's Prototype shown in Figure 534 , which is loosely inspired by Lemarchand's box from Clive ${ }_{535}$ Barker's novella The Hellbound Heart [23].

${ }_{536}$ In our bimodal game, a box and four wheels that are ar${ }_{537}$ ranged symmetrically in the center of the screen are rotated 538 using touch, and symbols are drawn using a stylus. The box 539 has a window in one corner that exposes symbols etched into 540 the wheel over which the window is oriented. Upon selecting 541 the desired wheel by rotating the box, the wheel itself is then 542 rotated by touch to select from its different symbols. Those 543 wheels not selected rotate automatically to ensure the player 544 is able to see all symbols in play. Once the desired symbol 545 is inside of the box's window, a player can draw the symbol 546 (gesture) anywhere on the screen. If a unistroke symbol is rec547 Ognized as the selected symbol within the window, and if there 548 is an enemy on the screen matching that symbol, then the en549 emy is banished to another realm. Enemies are arms that grope 550 their way towards the box, and once close enough, shoot light551 ning (or "magic") at the box in order to wreak havoc. As men552 tioned, each arm has a symbol on its hand which matches one 553 of the symbols on one of the four wheels. The associated sym554 bol on the wheel is highlighted lightly once the corresponding $555 \mathrm{arm}$ is visible on the screen to assist the player with locating 556 matches. When a gesture is recognized and the enemy is ban557 ished, a new symbol appears on the wheel; this continues until 558 the game ends. At the start of a round, a player has approxi559 mately 10-14 seconds to banish an enemy before it reaches the 560 cube and is able to attack. Similarly, there is a sufficient delay 561 between when new enemies are created so that the game pace is 
${ }_{562}$ leisurely. As the game progresses, enemies are generated faster 563 and move faster (approximately 3-6 seconds to reach the box) 564 So that not all arms can be defeated before they begin to attack. ${ }_{565}$ At this time, players are forced to react as quickly as possible 566 in order to defend the box before too much damage is taken. ${ }_{567}$ By the end, there are usually six to twelve enemies attacking at 568 once.

569 The Lemarchand's Prototype gesture set comprises the 40 570 unistroke symbols shown in Figure 6, which were inspired by 571 different sources. The star and right arrow variants, unistroke 572 ' $\mathrm{X}$ ', caret, check, pigtail variant, and curly and square brack573 ets were derived from \$1-GDS [1], which we extended with 574 the rounded brackets. The ' 3 ', ' 6 ', ' 8 ', ' $a$ ', ' $g$ ', 'm', and tri575 angle variant were derived from $\operatorname{EDS} 1$ [19]. The ' $\alpha$ ', rect576 angle (square), heart, curly, '?', and spiral variant are due to 577 EDS 2 [19]. The '2', '@', 'b', 'e', 'h', 'q', and 'y' gestures 578 come from UJI [20]. We also included the division gesture 579 (second row, second column in figure) from the Graffiti [24] 580 gesture set. Finally, from no particular source, we also added ${ }_{581}$ the ' $\&$ ', ' $\infty$ ', ' $\Sigma$ ', ' $\sigma$ ', ' $\sim$ ', transistor, and register symbols.

\section{5.1. Experimental Design}

583 Since our evaluation involved a large number of direction 584 sensitive unistroke gestures, we were only concerned with rec585 ognizers designed to solve this problem. $1^{\phi}$ generally does not 586 perform well when gestures are symmetric or rotation variant; ${ }_{587}$ Protractor is considered to be a $\$ 1$ enhancement ${ }^{2}$ because of its 588 speedup and similar (sometimes better) accuracy; $\$ \mathrm{~N}$ as well 589 as \$N-Protractor are extensions designed to also handle multi590 stroke gestures; and \$P is a general purpose, stroke order and 591 direction invariant recognizer. Following the cheatsheet pro592 vided by Vatavu et al. [6], we evaluated our recognizer along593 side Protractor because of its support for stroke direction sen594 sitivity, low coding complexity, and low algorithmic complex595 ity. For the in game Protractor recognizer, we integrated the ${ }_{596}$ reference source code available on the $\$ 1$ project website ${ }^{2}$. The 597 standard way of evaluating \$-family recognizers is to increment 598 the template count from 1 template per gesture to the maximum 599 available (usually 9 or 10). In practice, however, a user is un600 likely to provide more than one example of each gesture [5], or 601 perhaps at most, two to three. Further, it would be quite time 602 consuming to have a participant play the game 10 times, once ${ }_{603}$ for each count of templates per gesture from one to ten, and ${ }_{604}$ performance would likely deteriorate as participants become 605 lethargic with repeated game play. As a compromise, we set606 tled on a 3-level within-subjects design for each recognizer and ${ }_{607}$ between-subjects design between recognizers. That is, partici${ }_{608}$ pants were asked to play the game three times utilizing one rec609 ognizer, each iteration with a different number of templates per ${ }_{610}$ gesture: 1, 3, and 10. Each recognizer was evaluated with 12 611 participants ( 24 total) recruited from the University of Central ${ }_{612}$ Florida, and each participant was compensated $\$ 10$ for approxi613 mately 65 minutes of time. Of the participants that utilized Pro${ }_{614}$ tractor, 10 were male ( 2 female), 10 were right handed ( 2 left),

\footnotetext{
${ }^{2}$ See the $\$ 1$ unistroke recognizer project website: https://depts. washington.edu/aimgroup/proj/dollar/
}

615 all but 1 had prior experience with pen and touch interfaces ( 2 616 had a lot of experience), and the ages ranged from 18-24 (av617 erage 21.3). Of the participants that utilized Penny Pincher, 7 618 were male ( 5 female), 9 were right handed ( 3 left), all but 2 had 619 prior experience with pen and touch interfaces (no had a lot of 620 experience), and the ages ranged from 14-34 (average 21.6).

621 5.1.1. Procedure

622 Each participant was first acquainted with the experiment 623 and then asked to provide 10 samples of each gesture. Our data 624 collection apparatus allows for five samples to be collected at 625 a time, each in a different area on the display. Above each lo626 cation, an example of the required gesture was shown. Further, 627 the order of the 400 samples (forty gestures times ten) were 628 randomly permuted. After the training data was collected, the ${ }_{629}$ participant filled out a pre-questionnaire to collect demographic 630 information. Next the participants played a tutorial round of the 631 game where all of the mechanics were introduced, though the ${ }_{632}$ recognizers were not active at this time.

${ }_{633}$ After the practice round was completed, a participant played 634 the game three times: with 1,3 , and 10 templates per ges635 ture loaded. However, the order was randomly permuted, and 636 since there were three levels, there were six possible permuta${ }_{637}$ tions. Having twelve participants, we ensured that each permu638 tation was tested twice. During a single iteration of the game, ${ }_{639}$ the order of the enemy gestures were randomly selected, and 640 each symbol appeared three times so that once the experiment 641 was completed, each gesture was attempted at least nine times. ${ }_{642}$ However, a single instance of a gesture can be attempted at most 643 three times. After three unsuccessful attempts, the associated 644 enemy is banished regardless. Note that the second and third 645 attempts were included to allow the participant additional prac${ }_{646}$ tice in case of bad form, though we only consider first attempts 647 when calculating accuracy.

\section{5.2. Baseline Results}

649 Using the training data only, we first estimated baseline 650 results - the highest accuracy possible when there are no ad${ }_{651}$ ditional stress factors to consider. Specifically, we examine two 652 scenarios: user dependent accuracy and user independent ac653 curacy. A user dependent scenario may represent a situation 654 where a user provides templates for gestures that only he or she 655 will write in the game. For example, this may occur when cus656 tom gestures are mapped to certain commands within a game, 657 or when user dependent recognition is expected to be important 658 as of game play. User independent recognition, on the other 659 hand, may be important in a variety of different scenarios such 660 as:

\section{1} 662

- A player borrows his friend's mobile device to try out a game trained with his friend's example gestures.

- A multiplayer game involves cooperative gesture creation and manipulation such that ad hoc gestures are shared amongst the players. 

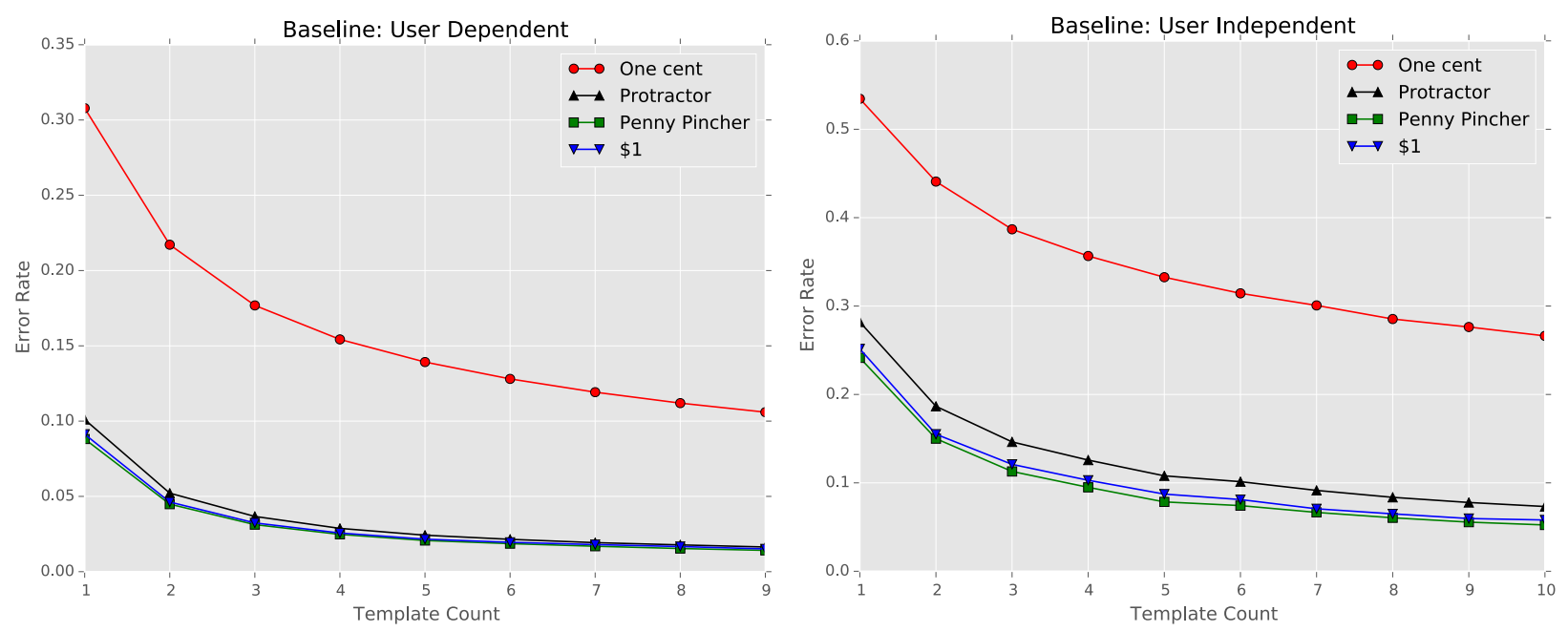

Figure 7: Baseline accuracy for user dependent (left) and user independent (right) test case scenarios based on training data as candidates and templates. Note that in the user dependent case, the template count only goes up to nine. This limit is because one sample per gesture is saved as a candidate gesture to be classified in each test, and there are only ten samples collected per gesture. 673 dard tests and candidate gestures are randomly 674 selected from the participant's training data. Results are shown 675 Figure 7. Although in game data (as discussed in the next sub676 section) was collected and evaluated for only two recognizers, 677 we ran this evaluation over all of the unistroke gesture rec678 ognizers. In the user dependent scenario, the $\$ 1$ and Penny ${ }_{679}$ Pincher recognizers perform almost identically and are the best ${ }_{680}$ performing recognizers. At one, two, and three templates per ${ }_{681}$ gesture, Penny Pincher achieves $8.8 \%, 4.5 \%$, and $3.1 \%$ recog${ }_{682}$ nition error rates, whereas $\$ 1$ is at $9.1 \%, 4.6 \%$, and $3.2 \%$; and 683 by ten templates per gestures, they fall to $1.4 \%$ and $1.5 \%$ re${ }_{684}$ spectively. Penny Pincher, therefore, sees only a very slight 685 advantage over $\$ 1$. Protractor is also very close, starting out a $68610.1 \%, 5.2 \%$, and $3.7 \%$, and drops to $1.6 \%$ for the same tem${ }_{687}$ plate counts, and $1^{\phi}$ is the worst performer overall with this ${ }_{688}$ particular dataset.

689 The relationship between the recognizers in user indepen${ }_{690}$ dent baseline results are similar, although, as expected, all recog691 nition error rates are worse due to differences in style and hand692 writing between participants. Again, $\$ 1$ and Penny Pincher ${ }_{693}$ achieve close results, with Penny Pincher being slightly bet694 ter. At one, two, and three templates per gesture, Penny Pincher 695 achieves a $24.1 \%, 15.0 \%$, and $11.3 \%$ recognition error rate, and 696 by ten templates per gesture, this drops to $5.2 \%$. $\$ 1$ 's recogni697 tion error rate is not as low, but still reaches $25.2 \%, 15.5 \%$, and $69812.1 \%$, and $5.8 \%$ for the same template counts. The third best 699 recognizer is Protractor, which falls slightly behind the others
700 at $28.2 \%, 18.7 \%$, and $14.6 \%$ for one, two, and three templates, 701 and $7.3 \%$ by ten templates per gesture.

${ }_{702}$ These initial baseline results suggest that Penny Pincher 703 should do as well or even slightly better than Protractor in the 704 game. Further, although $\$ 1$ was not explicitly evaluated in the 705 game, Penny Pincher should perform almost identically.

\section{5.3. In Game Results}

707 The in game recognition error rates for Protractor and Penny ros Pincher are shown in Table 3. Considering baseline results, we 709 noticed that Protractor and Penny Pincher were further apart in 710 recognition error rates than expected. Upon further inspection, 711 we found that the reference source ${ }^{2}$ scales strokes for Protractor 712 as well as $\$ 1$. However, this is not necessary with our dataset, 713 and in fact, performance is much better without scaling. There714 fore, we also include results for the in game data using Protrac715 tor without scaling, and further discussions refer to this latter 716 result. It can be seen that the Penny Pincher recognition error 717 rates are superior for all levels, where the error rate is decreased 718 by $16.1 \%, 21.8 \%$, and $10.5 \%$ for one, three, and ten templates 719 per gesture respectively.

720 For the remaining tests in this subsection, we used samples ${ }_{721}$ collected during game play to evaluate user dependent and user 722 independent scenarios for Penny Pincher, $\$ 1$, Protractor and $1^{\phi}$. ${ }_{723}$ We utilize this configuration to ensure that all recognizers are

\begin{tabular}{|c||c|c|c||c|c|}
\hline \multicolumn{1}{|c||}{} & \multicolumn{3}{c||}{ Protractor } & \multicolumn{2}{c|}{ Penny Pincher } \\
\hline$\#$ & Baseline & Game & Without Scaling & Baseline & Game \\
\hline 1 & $11.9 \%$ & $34.4 \%$ & $27.4 \%$ & $8.8 \%$ & $23.0 \%$ \\
3 & $4.6 \%$ & $24.4 \%$ & $22.4 \%$ & $3.1 \%$ & $17.5 \%$ \\
10 & $2.1 \%$ & $19.0 \%$ & $13.3 \%$ & $1.4 \%$ & $11.9 \%$ \\
\hline
\end{tabular}

Table 3: Summary of baseline and in game recognition error rates for Protractor and Penny Pincher for 1, 3, and 10 templates per gesture. Because the in game protractor implementation scales strokes, we also report the error rates without scaling, which in our tests are much lower. 

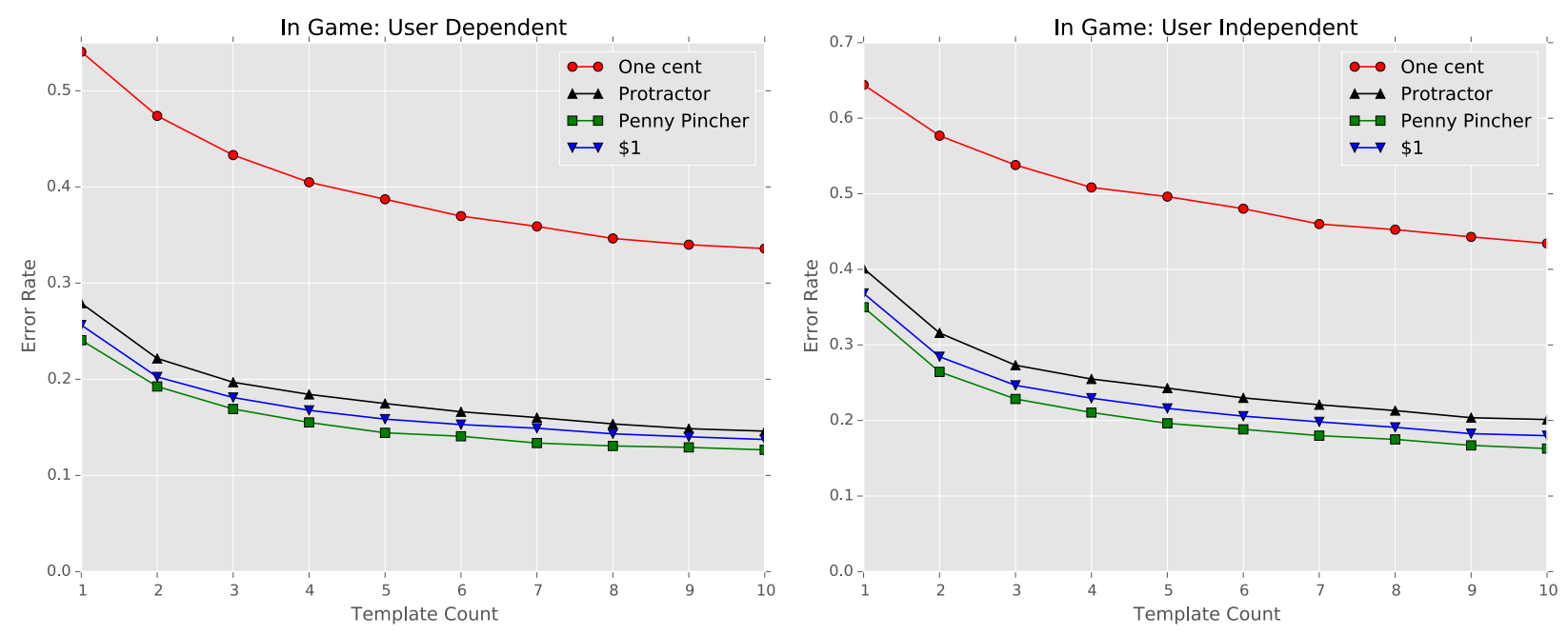

Figure 8: Recognition error rate of user dependent (left) and user independent (right) test case scenarios using the first in game gesture attempts as the candidate gestures and training data as the template gestures.

724 evaluated with the same distribution of online samples. The 725 experimental setup is similar to the standard test (Section 4.3) 726 except that the candidate gestures are any of those gestures that ${ }_{727}$ were drawn during game play by any participant. Since there 728 are 24 participants and 9 first attempts of each gestures, there 729 are 216 candidates per gesture to choose from in each of the 7301000 iterations. Further, recall that the template gestures are ${ }_{731}$ chosen at random from the training data in each iteration.

732 With the in game data, the difference between Penny Pincher ${ }_{733}$ and $\$ 1$ is more pronounced than in the baseline test, see Figure 734 8. With one, two, and three templates, Penny Pincher achieves 735 recognition errors rates of $24.1 \%, 19.2 \%$, and $16.9 \%$ respec736 tively, which drops to $12.7 \%$ by ten templates. $\$ 1$ sees $25.6 \%$, ${ }_{737} 20.2 \%$, and $18.1 \%$, which then drops to $13.7 \%$ for the same 738 template counts respectively. This represents a $5.8 \%, 4.9 \%$, and ${ }_{739} 6.6 \%$ reduction in error rate for the first three template counts, 740 and a $7.3 \%$ reduction for ten templates. Protractor is slightly 741 less accurate than $\$ 1: 27.9 \%, 22.2 \%$, and $19.7 \%$, which then 742 drops to $14.6 \%$. Compared to the baseline test results, the in ${ }_{743}$ game accuracy of Penny Pincher dropped by $16 \%$ at one tem${ }_{744}$ plate per gesture and $11.6 \%$ at nine templates per gesture in the 745 user dependent test. $\$ 1$ accuracy also dropped by $18.2 \%$ and $74612.6 \%$ for the same template counts. So, although in the user ${ }_{747}$ dependent baseline tests, Penny Pincher and $\$ 1$ were close in 748 their recognition errors rates, $\$ 1$ had a larger overall drop with 749 the in game data. Protractor's drop was even more dramatic, $75021.9 \%$ and $14.5 \%$.

751 When looking at the user independent test results, it can 752 be seen that the trends are very similar. Penny Pincher, again, 753 is the best achiever in recognition errors rates: $35.0 \%, 26.4 \%$, 754 and $22.8 \%$ for one, two, and three templates per gesture, which 755 drops to $16.3 \%$ by ten templates. This is followed by $\$ 1: 36.8 \%$, ${ }_{756} 28.4 \%$, and $24.7 \%$ for the lower template counts, and $18.0 \%$ for 757 ten templates. Protractor is not too far behind at $40.1 \%, 31.6 \%$, ${ }_{758} 27.3 \%$, and $20.1 \%$ for the same four template counts. Between 759 Penny Pincher and \$1, our recognizer enjoys a 5.1\%, 7.5\%, and ${ }_{760} 8.3 \%$ reduction in error for up to three templates per gesture,
761 and by ten, the reduction reaches $10.4 \%$. It is worth noting that 762 the margin between $\$ 1$ and Penny Pincher is somewhat con763 sistent in all of the charts (except the baseline user dependent 764 test) as the template counts increase. This implies the percent765 age reduction in error rate continues to improve as the template 766 count increases, even if the difference in accuracy is somewhat 767 constant.

\section{5.4. Misclassified Gestures}

769 In a game where one has to quickly find a symbol, mechan770 ically arrange components through touch, and then rapidly pen 771 a gesture, mistakes are going to be made. This is supported by 772 the fact that our baseline results are dramatically different from 773 the in-game results, as is often the case [21, 22]. However, it 774 is also important to understand the cause of these errors. In 775 a post analysis of first attempt misclassifications, we visually 776 examined each recorded sample that was classified incorrectly 777 and further divided all errors into four categories: misfire, mis778 taken, malformed, and false negative. A misfire occurs when 779 one accidentally strikes the pen against the interactive display 780 or when one starts, but does not complete a gesture. One cause ${ }_{781}$ of this may be that the participant realized that he or she was 782 about to pen an incorrect gesture. Switching between touch and ${ }_{783}$ pen may be another cause of misfires, and in a couple of cases, ${ }_{784}$ participants tried to rotate the box or a wheel with the stylus. 785 To determine misfires, we assumed that the symbol framed by 786 the selection window was the gesture attempted. It is possi787 ble that a participant performed a gesture without first selecting 788 the correct symbol, and though we do not have a method to 789 differentiate this situation, it does not seem apparent upon vi790 sual inspection of the collected gestures. A mistaken gesture 791 occurs when one gesture is expected but a different gesture is 792 drawn. A common example of this occurred when a partici793 pant should have drawn an '@' gesture, but he or she drew a ${ }_{794}$ spiral instead. These errors may have been caused by rushed 795 game play, where the participant did not properly observe the 


\begin{tabular}{|l||c|c||c|c|}
\hline \multicolumn{1}{|c||}{} & \multicolumn{2}{c||}{ Protractor } & \multicolumn{2}{c|}{ Penny Pincher } \\
\hline$\#$ & Count & Percentage & Count & Percentage \\
\hline Misfire & 118 & $10.5 \%$ & 121 & $15.8 \%$ \\
Mistaken & 71 & $6.3 \%$ & 56 & $7.3 \%$ \\
Malformed & 517 & $45.8 \%$ & 329 & $43.1 \%$ \\
False Negative & 423 & $37.5 \%$ & 258 & $33.8 \%$ \\
\hline Total & 1129 & - & 764 & - \\
\hline
\end{tabular}

839 vectors. This immediately afforded us two benefits, that can840 didate gestures neither need to be scaled nor do they have to 841 be translated to a common origin for comparison. Second, we 842 worked to eliminate the need for normalization during template 843 matching. This is accomplished by normalizing the between844 point vectors of template gestures during training, and by as845 suming that the distance between points of resampled strokes

Table 4: Distribution of misclassification error types for first attempt gestures of in game Protractor and Penny Pincher results. Note that there were 4320 first attempts per recognizer: 12 participants by 40 gestures by 9 instances.

796 expected gesture and mistook, by broad shape similarity, a dif797 ferent gesture. Malformed gestures occur when the stroke is 798 readable - a human can recognize the symbol—but the form is 799 wrong; one example of this is when a player draws an $\alpha$ symbol 800 backwards, starting at the wrong end of the stroke. The $\$ \mathrm{~N}$ and ${ }_{801}$ \$P recognizers can handle this situation, but in our game, we do 802 not allow stroke direction invariance. Finally, a false negative is 803 simply a well formed gesture that is confused and misclassified 804 as another.

805 Note that the distinction between malformed gestures and 806 false negatives can be subjective. For instance, samples that 807 are human readable but excessively sloppy or misshaped are 808 classified as malformed, though there is no formal criteria. It 809 is also important to understand that a malformed gesture can 810 be correctly recognized, especially when the gesture exists in a ${ }_{811}$ "sloppiness space" [25] where its form is sufficiently far from 812 neighboring gestures and sloppiness is less problematic, which ${ }_{813}$ in part depends on the recognizer's internal representation of 814 gestures.

${ }_{815}$ The tabulated results are shown Table 4. Misfired and mis816 taken gestures were similar for both Protractor and Penny Pinche ${ }_{817}$ in raw numbers. This is not surprising given that participants 818 were under the same level of stress and their interaction with ${ }_{819}$ the game was expected to be similar. On the other hand, we 820 noticed that malformed gestures and false negatives were sig${ }_{821}$ nificantly higher with Protractor (with the in-game Protractor 822 recognizer variant). However, the percentage distribution be${ }_{823}$ tween malformed and false negative gestures is almost identical 824 for both recognizers. As mentioned before, we expected partici825 pants (on average) to utilize both recognizers identically; there826 fore, this result is not surprising given that sloppiness is likely ${ }_{827}$ uniform, and because Protractor appears to be less accurate with 828 this particular dataset, the raw number of misclassifications are 829 accordingly higher without changing the distribution. The false 830 negative and malformed gesture counts would likely have been ${ }_{831}$ lower with Protractor's scaling removed, as previously noted. ${ }_{832}$ Though, despite a high number of misclassifications, especially ${ }_{833}$ with Protractor, users did not report being frustrated while play834 ing the game, and most enjoyed the challenge.

\section{${ }_{835}$ 6. Discussion and Future Work}

836 Penny Pincher was designed to be as fast as possible and we ${ }_{837}$ took a number of steps to achieve this goal. First, we decided to ${ }_{838}$ base our similarity metric on the dot product of between-point

846 are equal. With this approach, we reduced the recognition task 847 down to just addition and multiplication. As a result, Penny ${ }_{848}$ Pincher is the simplest and easiest to understand of all \$-family 849 recognizers. In terms of speed, only $1^{\phi}$ is faster, but as was 850 demonstrated, it seems that $1^{\phi}$ is not well suited for general 851 purpose gesture recognition. In terms of accuracy, for three ${ }_{852}$ datasets we achieved the lowest user independent recognition 853 error with accuracies between $97.5 \%$ and $99.9 \%$. Over an addi854 tional three datasets, our recognizer still remained competitive, 855 indicating that Penny Pincher is an excellent general purpose ${ }_{856}$ recognizer, even without considering speed. In one case, the 857 dataset was a multistroke dataset having varying stroke orders 858 and directions, which is why Penny Pincher did not perform as 859 well as \$N and \$N-Protractor with only a few templates loaded.

Computational performance, however, was initially our pri${ }_{361}$ mary concern. Given a difficult time budget, we worked to 862 develop a recognizer that could process as many templates as ${ }_{863}$ possible to achieve the highest recognition rate possible within 864 the specified constraint. In the budget portion of our evaluation 865 we demonstrated that Penny Pincher succeeded in this objec866 tive. With our test apparatus, Penny Pincher is able to process 867 approximately 30.3 templates per microsecond, whereas Pro868 tractor (generally the second most accurate recognizer in the 869 budget test) is limited to 5.7. Therefore, our recognizer is able 870 to achieve a percentage error that is less than $1 \%$ for all but one 871 of the tested datasets given as little as $60 \mu \mathrm{s}$. When consider872 ing reduction in recognition error for the various time budgets, 873 Penny Pincher sees an $83 \%$ or higher reduction in most cases, 874 and in several instances, the reduction is $94 \%$ or more. The ${ }_{875}$ UJI dataset is by far the most difficult to work with because of 876 the similarity in gestures (e.g, upper- vs lower-case ' $O$ ') and 877 because the number of gesture classes is large (97). However, 878 the reduction in error still ranges between $26 \%$ and $31 \%$. This 879 indicates that from all of the $\$$-family recognizers, ours is best 880 suited for tight time budgets, when a large number of samples 881 are available. Also note that our budgets were selected because 882 of the number of examples available in the datasets and because 883 of our apparatus. In other environments, such as an implemen884 tation in an interpreted language running on a mobile device, 885 budgets would likely have to be much higher to attain the same 886 accuracies.

887 Our derivation of the recognizer assumes that the between888 point vectors are of equal length. While this is not strictly true, 889 the empirical probability distribution of relative between-point 890 vector lengths shows that as an approximation the assumption is 891 valid. But because the distribution is left skewed, what does this 892 really mean for shorter vectors with respect to pattern match893 ing? In a resampled stroke, between-point vectors on straight 894 lines will have the longest length. Relative to shorter vectors 895 that fall on cusps, straight lines will have a larger influence 
896 on the final estimate. Therefore, under our assumption, Penny ${ }_{897}$ Pincher winds up weighting straight lines with greater impor898 tance. Interestingly, in informal ad hoc testing, we saw that this 899 had a positive impact on accuracy and as part of future work, 900 it is worth investigating further to determine how lines, curves, 901 and cusps impact accuracy with respect to our method.

902 We were surprised to see Penny Pincher perform so well as 903 compared to the other recognizers when testing involved lower 904 template counts. However, the majority of datasets evaluated 905 were collected in a relaxed stress-free lab environment. This 906 made us curious about how well our recognizer could do in a 907 more difficult setting, again using a small number of templates 908 as often occurs in practice, which is why we developed Lemarc909 hand's Prototype - to evaluate the effectiveness of Penny Pincher 910 in a stressful game setting, one in which users are likely to be ${ }_{911}$ less precise in their writing. As it turns out, Penny Pincher 912 still performs remarkably well. Compared to $\$ 1$ and Protrac913 tor, the average recognition error rates were lower with a $5.8 \%$ 914 to $10.4 \%$ reduction in error rate. Even though in the user de915 pendent test, Penny Pincher scored just slightly better than its 916 closest competitor, the difference was still more pronounced in 917 the user independent tests. These results in combination with 918 previous tests suggest that Penny Pincher is a good general rec${ }_{919}$ ognizer for small and large datasets, and that because of its sim920 plicity, our recognizer will likely be preferred by many for its 921 speed, quick implementation, and non-sacrificing accuracy.

922 In our evaluations we demonstrated the benefits of Penny ${ }_{923}$ Pincher, but it is also important to understand its various limita${ }_{924}$ tions. Since we compare between-point vectors, Penny Pincher ${ }_{925}$ is scale invariant, though unlike other recognizers it is not rota${ }_{926}$ tion invariant. As is turns out though, with the datasets we eval${ }_{927}$ uated, this was not an issue and with more templates loaded, 928 variations in angular displacement are well represented. Our ${ }_{929}$ recognizer is also not intrinsically a multistroke recognizer. Sim${ }_{930}$ ilar to $\$ \mathrm{~N}$, we concatenate multiple strokes together to create a ${ }_{931}$ single unistroke gesture for template matching, but unlike $\$ \mathrm{~N}$, ${ }_{932}$ we do not generate all of a sample's various permutations. In${ }_{933}$ stead, training Penny Pincher with additional examples may es${ }_{934}$ sentially have the same effect and therefore, it may be unneces${ }_{935}$ sary to derive the numerous permutations (some of which may ${ }_{936}$ never occur in practice anyways) to achieve high accuracy. This ${ }_{937}$ is supported, in part, by the fact that we achieve over $99 \%$ ac${ }_{938}$ curacy in the budget test with the MMG dataset, and Penny ${ }_{939}$ Pincher also achieves high accuracy (relative to $\$ \mathrm{~N}$ and $\$ \mathrm{~N}$ ${ }_{940}$ Protractor) with UJI.

${ }_{941}$ The idea behind our work is that there are potentially hun${ }_{942}$ dreds of templates available per gestures for the recognizer to ${ }_{943}$ utilize, but this poses two possible problems. First, a large ${ }_{944}$ amount of memory is required to store high counts of tem${ }_{945}$ plate data. Suppose that an implementation is using double${ }_{946}$ precision floating point numbers that are 8 bytes long. Each ${ }_{947}$ two-dimensional point then requires 16 bytes and with a six948 teen point resampling count, each template requires 256 bytes 949 of memory (not including any overhead needed for the template 950 structure itself). Given sixteen gestures and two-hundred sam${ }_{951}$ ples per gesture, approximately $800 \mathrm{KiB}$ of memory are needed 952 to store all of the data. For most systems, $800 \mathrm{KiB}$ is negligi-
953 ble, but in some cases this may be impractical. The second is954 sue is that in certain applications there are simply not that many 955 examples available. This will occur, for instance, if a user is ${ }_{956}$ supplying custom gestures to a user interface. As we saw in 957 the evaluation though, Penny Pincher provides high accuracy 958 already with even a small number of templates. Further, over 959 time as the system is used, additional examples can be collected 960 and the recognizer can be continuously retrained with new data. 961 As part of future work, in the spirit of making things go 962 fast, it would be interesting to look at alternatives to equidis963 tant resampling of candidate gestures. With thousands of tem964 plates loaded, the majority of time is spent in matching, but by 965 eliminating the last remaining geometric library calls (namely $966 s q r t$ ), there may be a small positive effect. More importantly 967 though, not all templates give relevant additional information 968 about the sample space. For example, if a minimum subset of 969 the available templates are sufficient to define a portion of the 970 sample subspace (so that if any candidate gesture falls within 971 this subspace, it will be classified correctly), then any addi972 tional templates in this subspace are superfluous. Therefore it 973 would be interesting to research template selection techniques 974 that are appropriate for Penny Pincher and that are also within 975 the theme of the \$-family. Finally, there are numerous ways in 976 which games utilizing gesture recognition can be imagined. In 977 order to further validate the effectiveness of Penny Pincher and 978 other recognizers in a game environment, additional studies are 979 warranted. One example includes a multi-player tabletop fight980 ing game in which four or more players are interacting and as 981 a result, numerous gestures are being produced at varying an982 gles and orientations, which is a potentially very challenging 983 scenario to handle.

\section{7. Conclusion}

${ }_{985}$ We have presented Penny Pincher, a fast and accurate \$986 family gesture recognizer that compares between-point vectors 987 to estimate similarity. This recognizer is stripped down to the 988 bare essentials so that after a gesture is resampled, only ele989 mentary arithmetic is required to achieve great performance. ${ }_{990}$ In a user independent evaluation utilizing six unique datasets 991 of varying complexity, we demonstrated that Penny Pincher 992 outperforms $\$ 1$, Protractor, $\$ \mathrm{~N}, \$ \mathrm{~N}$-Protractor, and $1^{\phi}$ in three 993 cases with just a small number of templates (achieving $97.5 \%$, $99499.8 \%$, and $99.9 \%$ accuracy), and remains competitive in the 995 other cases. In a budget test where each recognizer is given a 996 limited amount of time to perform its recognition task, Penny 997 Pincher significantly outperforms the other recognizers, achiev998 ing a reduction in recognition error of between $83 \%$ and $99 \%$ 999 with four datasets, and a $31 \%$ reduction with the most diffi000 cult dataset. Finally, with an online user evaluation of Penny ${ }_{001}$ Pincher and Protractor in a video game, we were able to demon1002 strate that Penny Pincher still yields higher accuracy. Utilizing ${ }_{1003}$ the in game data we collected, we also evaluated $\$ 1$ and $1^{\phi}$, 004 varying template counts, as well as the user independent and 005 user dependent use case scenarios. In all tests, Penny Pincher 1006 achieved the highest accuracy with our dataset. 


\section{8. Acknowledgements}

This work is supported in part by NSF CAREER award IIS${ }_{1009} 0845921$ and NSF award CCF-1012056. Also, we would like 1010 to thank the members of ISUE lab for their support and the 1011 anonymous reviewers for their useful comments and feedback.

\section{Appendix A. Penny Pincher Pseudocode}
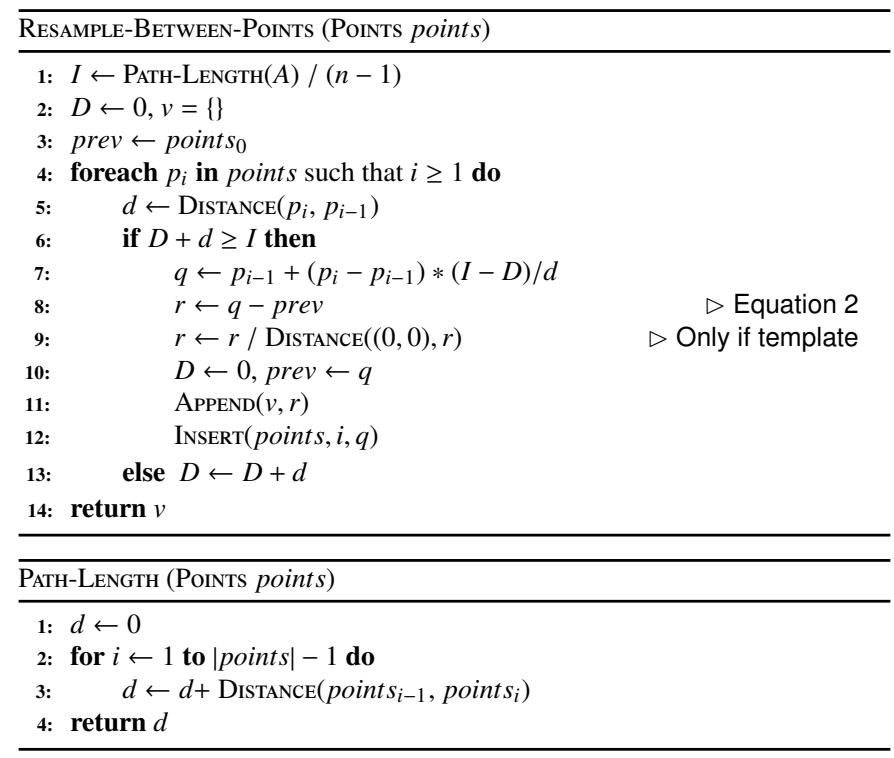

\begin{tabular}{l} 
Distance $($ PoInT $a$, PoInT $b)$ \\
1: return $\sqrt{\left(a_{x}-b_{x}\right)^{2}+\left(a_{y}-b_{y}\right)^{2}}$ \\
\hline
\end{tabular}

\begin{tabular}{ll}
\hline Recognize (Points $g$, Templates $\mathcal{T}$ ) \\
\hline 1: & $c \leftarrow$ Resample-Between-Points $(g)$ \\
2: & similarity $\leftarrow-\infty$ \\
3: & foreach $t$ in $\mathcal{T}$ do \\
4: & $d \leftarrow 0$ \\
5: & for $i \leftarrow 0$ to $n-2$ do \\
6: & $\quad d \leftarrow d+t_{i_{x}} c_{i_{x}}+t_{i_{y}} c_{i_{y}}$ \\
7: & if $d>$ similarity then \\
8: & similarity $\leftarrow d$ \\
9: & $T \leftarrow t$ \\
10: & return $\langle T$, similarity $\rangle$ \\
\hline
\end{tabular}

\section{${ }_{1013}$ References} Wobbrock JO, Wilson AD, Li Y. Gestures without libraries, toolkits or 1085 the 20th Annual ACM Symposium on User Interface Software and Technology. UIST '07; New York, NY, USA: ACM. ISBN 978-1-59593-6790; 2007, p. 159-68. URL: http://doi .acm.org/10.1145/1294211. 1294238. doi:10.1145/1294211.1294238.

2] Anthony L, Wobbrock JO. A lightweight multistroke recognizer for user interface prototypes. In: Proceedings of Graphics Interface 2010. GI '10; Toronto, Ont., Canada, Canada: Canadian Information Processing Society. ISBN 978-1-56881-712-5; 2010, p. 245-52.

[3] Anthony L, Wobbrock JO. \$n-protractor: A fast and accurate multistroke recognizer. In: Proceedings of Graphics Interface 2012. GI '12; Toronto, Ont., Canada, Canada: Canadian Information Processing Society. ISBN 978-1-4503-1420-6; 2012, p. 117-20. URL: http: //dl.acm.org/citation.cfm?id=2305276. 2305296.
[4] Herold J, Stahovich TF. The 1\&cent; recognizer: A fast, accurate, and easy-to-implement handwritten gesture recognition technique. In: Proceedings of the International Symposium on Sketch-Based Interfaces and Modeling. SBIM '12; Aire-la-Ville, Switzerland, Switzerland: Eurographics Association. ISBN 978-3-905674-42-2; 2012, p. 39-46. URL: http: //dl.acm.org/citation. cfm?id=2331067. 2331074.

5] Li Y. Protractor: A fast and accurate gesture recognizer. In: Proceedings of the SIGCHI Conference on Human Factors in Computing Systems. CHI '10; New York, NY, USA: ACM. ISBN 978-1-60558-929-9; 2010, p. 2169-72. URL: http://doi .acm.org/10.1145/1753326.1753654. doi:10.1145/1753326.1753654.

6] Vatavu RD, Anthony L, Wobbrock JO. Gestures as point clouds: A $\$ p$ recognizer for user interface prototypes. In: Proceedings of the 14th ACM International Conference on Multimodal Interaction. ICMI '12; New York, NY, USA: ACM. ISBN 978-1-4503-1467-1; 2012, p. 273-80. URL: http://doi .acm.org/10.1145/2388676.2388732. doi:10.1145/2388676.2388732

7] Rubine D. Specifying gestures by example. SIGGRAPH Computer Graphics 1991;25(4):329-37. URL: http://doi.acm.org/10.1145/ 127719.122753. doi:10.1145/127719.122753.

8] Taranta II EM, LaViola Jr. JJ. Penny pincher: A blazing fast, highly accurate \$-family recognizer. In: Proceedings of the 41st Graphics Interface Conference. GI '15; Toronto, Ont., Canada, Canada: Canadian Information Processing Society. ISBN 978-0-9947868-0-7; 2015, p. 195-202. URL: http://dl . acm.org/citation. cfm?id=2788890.2788925.

9] Gregory J. Game Engine Architecture. Ak Peters Series; Taylor \& Francis; 2009. ISBN 9781568814131. URL: http://books .google.com/ books?id=LJ20tsePKk4C.

10] Gray WD, Boehm-Davis DA. Milliseconds matter: An introduction to microstrategies and to their use in describing and predicting interactive behavior. Journal of experimental psychology: Applied 2000;6:322-35. doi:10.1037//1076-898X.6.4.322

1] Press WH, Teukolsky SA, Vetterling WT, Flannery BP. Numerical Recipes in C (2Nd Ed.): The Art of Scientific Computing. New York, NY, USA: Cambridge University Press; 1992. ISBN 0-521-43108-5.

] Reaver J, Stahovich TF, Herold J. How to make a quick\$: Using hierarchical clustering to improve the efficiency of the dollar recognizer. In: Proceedings of the Eighth Eurographics Symposium on Sketch-Based Interfaces and Modeling. SBIM '11; New York, NY, USA: ACM. ISBN 978-1-4503-0906-6; 2011, p. 103-8. URL: http://doi .acm.org/10. 1145/2021164.2021183. doi:10.1145/2021164. 2021183.

13] Zhang Y, Deng W, Song H, Wu L. A fast pen gesture matching method based on nonlinear embedding. In: Tan T, Ruan Q, Chen X, Ma H, Wang L, editors. Advances in Image and Graphics Technologies; vol. 363 of Communications in Computer and Information Science. Springer Berlin Heidelberg. ISBN 978-3-642-37148-6; 2013, p. 22331. URL: http://dx.doi.org/10.1007/978-3-642-37149-3_27. doi:10.1007/978-3-642-37149-3_27.

14] Hwang Y, kap Ahn H. Convergent bounds on the euclidean distance. In: Shawe-taylor J, Zemel R, Bartlett P, Pereira F, Weinberger K, editors. Advances in Neural Information Processing Systems 24. 2011, p. 388-96. URL: http://books.nips.cc/papers/files/nips24/NIPS2011_ 0286.pdf.

] Hwang Y, Han B, Ahn HK. A fast nearest neighbor search algorithm by nonlinear embedding. In: 2012 IEEE Conference on Computer Vision and Pattern Recognition (CVPR). 2012, p. 3053-60. doi:10.1109/ CVPR. 2012.6248036.

[16] Vatavu RD. 1f: One accessory feature design for gesture recognizers. In: Proceedings of the 2012 ACM International Conference on Intelligent User Interfaces. IUI '12; New York, NY, USA: ACM. ISBN 9781-4503-1048-2; 2012, p. 297-300. URL: http://doi.acm.org/10. 1145/2166966.2167022. doi:10.1145/2166966.2167022.

[17] Fuccella V, Costagliola G. Unistroke gesture recognition through polyline approximation and alignment. In: Proceedings of the 33rd Annual ACM Conference on Human Factors in Computing Systems. CHI '15; New York, NY, USA: ACM. ISBN 978-1-4503-3145-6; 2015, p. 33514. URL: http://doi.acm.org.ezproxy.net.ucf.edu/10.1145/ 2702123.2702505. doi:10.1145/2702123.2702505.

[18] Almaksour A, Anquetil E, Quiniou S, Cheriet M. Personalizable penbased interface using lifelong learning. In: 2010 International Conference on Frontiers in Handwriting Recognition (ICFHR). 2010, p. 188-93. 
1100 [19] Vatavu RD, Vogel D, Casiez G, Grisoni L. Estimating the perceived dif1101 ficulty of pen gestures. In: Proceedings of the 13th IFIP TC 13 Inter1102 national Conference on Human-computer Interaction - Volume Part II. 1103 INTERACT'11; Berlin, Heidelberg: Springer-Verlag. ISBN 978-3-6421104 23770-6; 2011, p. 89-106. URL: http://dl.acm.org/citation. $1105 \quad \mathrm{cfm}$ ?id=2042118.2042130.

106 [20] Llorens D, Prat F, Marzal A, Vilar JM, Castro MJ, Amengual JC, et al. 1107 The ujipenchars database: a pen-based database of isolated handwritten 1108 characters. In: Calzolari N, Choukri K, Maegaard B, Mariani J, Odijk 1109 J, Piperidis S, et al., editors. Proceedings of the Sixth International Con1110 ference on Language Resources and Evaluation (LREC'08). Marrakech, 1111 Morocco: European Language Resources Association (ELRA). ISBN 1112 2-9517408-4-0; 2008,Http://www.lrec-conf.org/proceedings/lrec2008/.

1113 [21] Cheema S, Hoffman M, LaViola JJ. 3d gesture classification with linear 1114 acceleration and angular velocity sensing devices for video games. Enter1115 tainment Computing 2013;4(1):11-24.

1116 [22] Taranta II EM, Simons TK, Sukthankar R, Laviola Jr. JJ. Exploring 1117 the benefits of context in $3 \mathrm{~d}$ gesture recognition for game-based vir1118 tual environments. ACM Transactions on Interactive Intelligent Systems $11192015 ; 5(1): 1: 1-1: 34$. URL: http://doi.acm.org/10.1145/2656345. 1120 doi:10.1145/2656345.

1121 [23] Barker C. The hellbound heart. HarperTorch; 1991. ISBN 0061002828. 1122 [24] PalmPilot Handbook. 1997.

1123 [25] Goldberg D, Richardson C. Touch-typing with a stylus. In: Proceedings 1124 of the INTERACT '93 and CHI '93 Conference on Human Factors in 1125 Computing Systems. CHI '93; New York, NY, USA: ACM. ISBN 01126 89791-575-5; 1993, p. 80-7. URL: http://doi.acm.org/10.1145/ $1127 \quad 169059.169093$. doi:10.1145/169059.169093. 


\section{Compare Between-Point Vectors}

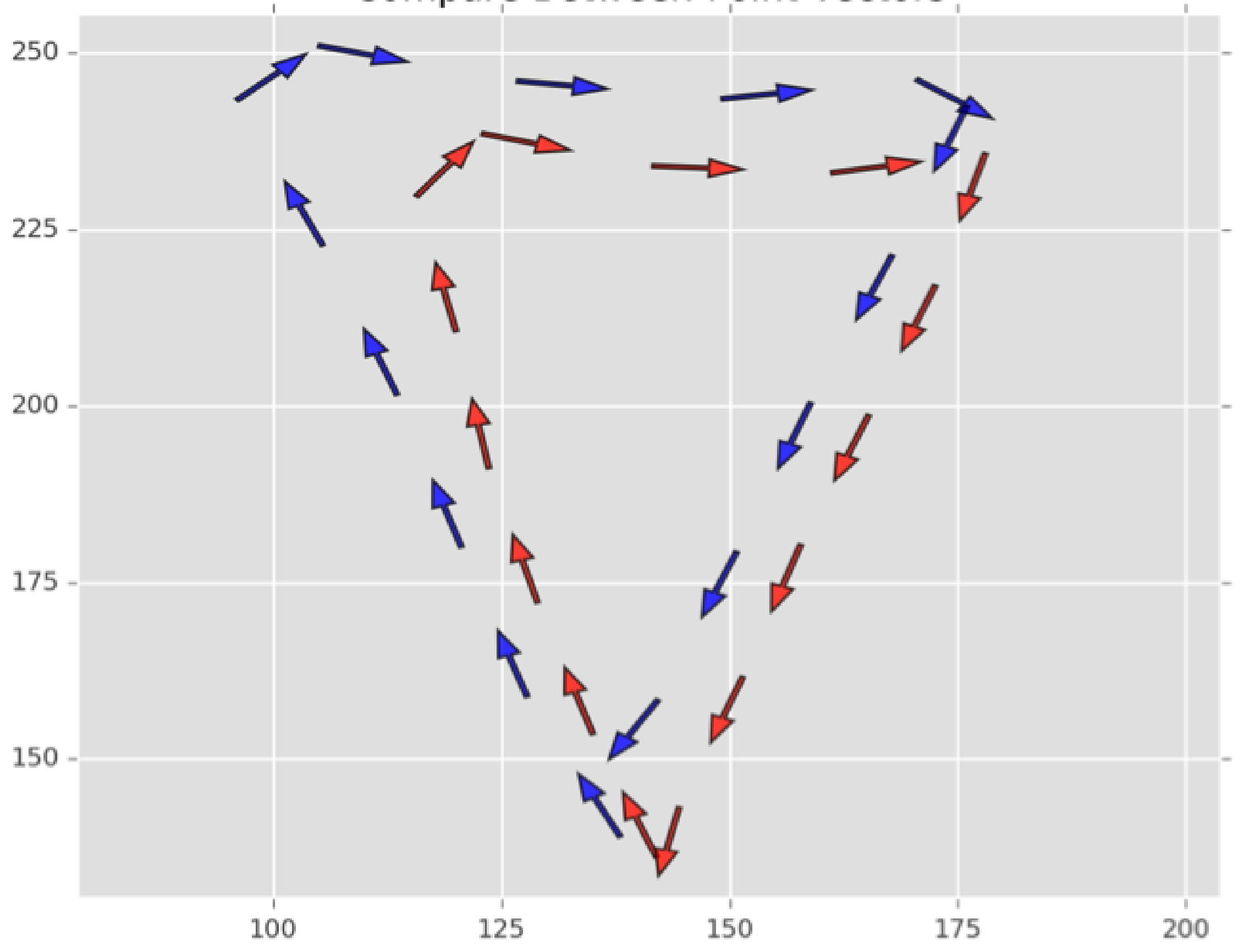

